\title{
Harnessing the Benefits of Neuroinflammation: Generation of Macrophages/Microglia with Prominent Remyelinating Properties
}

\author{
${ }^{\circledR}$ Manoj Kumar Mishra, ${ }^{1}$ Khalil S. Rawji, ${ }^{1}$ Michael B. Keough, ${ }^{1}$ Janson Kappen, ${ }^{1}$ Reza Dowlatabadi, ${ }^{2}$ \\ Hans J. Vogel, ${ }^{2}{ }^{\circledR}$ Sameeksha Chopra, ${ }^{3,4}{ }^{\circledR}$ Félix Distéfano-Gagné, ${ }^{5}$ Antoine Dufour, ${ }^{3,4}$ David Gosselin, ${ }^{5}$ and \\ (1)V. Wee Yong ${ }^{1}$ \\ ${ }^{1}$ Hotchkiss Brain Institute and Department of Clinical Neurosciences, ${ }^{2}$ Department of Biological Sciences, ${ }^{3}$ Department of Physiology \& \\ Pharmacology, ${ }^{4}$ McCaig institute for Bone and Joint Health, University of Calgary, Calgary, Alberta T2N 4N1, Canada, and ${ }^{5}$ Department of \\ Molecular Medicine, Université Laval, Quebec City, Quebec K2P 0J6, Canada
}

Excessive inflammation within the CNS is injurious, but an immune response is also required for regeneration. Macrophages and microglia adopt different properties depending on their microenvironment, and exposure to IL4 and IL13 has been used to elicit repair. Unexpectedly, while LPS-exposed macrophages and microglia killed neural cells in culture, the addition of LPS to IL4/IL13-treated macrophages and microglia profoundly elevated IL10, repair metabolites, heparin binding epidermal growth factor trophic factor, antioxidants, and matrix-remodeling proteases. In C57BL/6 female mice, the generation of $\mathrm{M}$ (LPS/IL4/IL13) macrophages required TLR4 and MyD88 signaling, downstream activation of phosphatidylinositol-3 kinase/ mTOR and MAP kinases, and convergence on phospho-CREB, STAT6, and NFE2. Following mouse spinal cord demyelination, local LPS/IL4/IL13 deposition markedly increased lesional phagocytic macrophages/microglia, lactate and heparin binding epidermal growth factor, matrix remodeling, oligodendrogenesis, and remyelination. Our data show that a prominent reparative state of macrophages/microglia is generated by the unexpected integration of pro- and anti-inflammatory activation cues. The results have translational potential, as the LPS/IL4/IL13 mixture could be locally applied to a focal CNS injury to enhance neural regeneration and recovery.

Key words: macrophages; microglia; neuroinflammation; oligodendrocyte; remyelination

Significance Statement

The combination of LPS and regulatory IL4 and IL13 signaling in macrophages and microglia produces a previously unknown and particularly reparative phenotype devoid of pro-inflammatory neurotoxic features. The local administration of LPS/IL4/ IL13 into spinal cord lesion elicits profound oligodendrogenesis and remyelination. The careful use of LPS and IL4/IL13 mixture could harness the known benefits of neuroinflammation to enable repair in neurologic insults.

Received July 27, 2020; revised Mar. 2, 2021; accepted Mar. 4, 2021.

Author contributions: M.K.M. and V.W.Y. designed research; M.K.M., K.S.R., M.B.K., J.K., R.D., S.C., F.D.-G., and D.G. performed research; M.K.M., K.S.R., M.B.K., J.K., R.D., H.J.V., S.C., F.D.-G., A.D., D.G., and V.W.Y. analyzed data; M.K.M. wrote the first draft of the paper; M.K.M., K.S.R., M.B.K., J.K., R.D., H.J.V., S.C., F.D.-G., A.D., D.G., and V.W.Y. edited the paper; M.K.M. and V.W.Y. wrote the paper.

M.K.M. was supported by Alberta Innovates-Health Solutions fellowship. K.S.R. held a Vanier Canada Graduate Scholarship and a studentship from the University of Calgary, Faculty of Medicine. M.B.K. previously received salary support from the Multiple Sclerosis Society of Canada and Alberta Innovates-Health Solutions. This work was supported by Multiple Sclerosis Society of Canada, Canadian Institutes of Health Research, and the Alberta Innovates-Health Solutions/CRI0 Team program operating grants. We thank Janet Wang and Yan Fan for skilled technical help.

The authors declare no competing financial interests.

Correspondence should be addressed to V. Wee Yong at vyong@ucalgary.ca.

https://doi.org/10.1523/JNEUROSCI.1948-20.2021

Copyright $\odot 2021$ the authors

\section{Introduction}

An excessive inflammatory response injures the nervous system, but neuroinflammation can also lead to beneficial outcomes (Yong et al., 2019; Guerrero and Sicotte, 2020; Rossi et al., 2020), including homeostatic neurogenesis (Ziv et al., 2006), protection against loss of axons and neurons (Hauben et al., 2000; Kipnis, 2016), and axonal regeneration (Yin et al., 2006; Zhou et al., 2020). Myelin repair (remyelination) is also facilitated by a properly directed inflammatory response (Lloyd and Miron, 2019; Rawji et al., 2020). Mice that lack both B and T lymphocytes (Bieber et al., 2003), and regulatory $\mathrm{T}$ cells (Dombrowski et al., 2017) have reduced spontaneous remyelination following demyelinating insults. Similarly, the removal of monocyte-derived macrophages with silica quartz dust (Triarhou and Herndon, 1985), clodronate liposomes (Kotter et 
al., 2005), or by genetic deletion of CCR2 that reduces their chemotaxis into lesion sites (Ruckh et al., 2012), impairs remyelination. Moreover, mice that are genetically deficient for prominent products of macrophages or of their CNS counterparts (microglia, including TNF- $\alpha$, IL1, or matrix metalloproteinase-9 [MMP-9]), display reduced remyelinating capacity (Wee Yong, 2010). Monocyte-derived macrophages from the circulation, border-associated macrophages that reside in the boundaries of the CNS (Kierdorf et al., 2019), and microglia have not been differentiated in many studies so they will be referred to as macrophages/microglia herein unless distinguished otherwise.

In support of the benefits of macrophages/microglia, approaches that increase their representation in lesions promote remyelination. These conditions include the stimulation of macrophages/microglia with drugs, such as amphotericin B or niacin (Doring et al., 2015; Rawji et al., 2020), the creation of a mechanical lesion within the spinal cord that mobilized immune cells (Foote and Blakemore, 2005), or the intraspinal transplantation of macrophages/microglia (Rapalino et al., 1998). Recently, it was reported that the persistent intracellular accumulation of myelin within phagocytes induced a lesionpromoting phenotype in demyelinating disorders; by boosting the efflux of myelin-associated lipids, this accelerated remyelination ex vivo and in vivo (Bogie et al., 2020). Also, the killing of impaired microglia and subsequent repopulation of pro-regenerative microglia was found to promote remyelination in mice (Lloyd et al., 2019).

Macrophages and microglia adopt different functional states depending on the activation stimuli (Wynn and Vannella, 2016). For example, LPS stimulation enhances phagocytic and cargo degradation capabilities (Cooper et al., 1984), which are important to remove tissue debris that can interfere with regenerative mechanisms in lesions (Vallieres et al., 2006; Church et al., 2017). Stimulation with immune cytokines IL4/IL13 have also frequently been associated with enhancements of repair properties of macrophages. These cytokines promote arginase-1 (Arg-1) expression, which catalyzes ornithine from arginine; the ornithine in turn generates polyamines for cell division, and proline for collagen synthesis (Rath et al., 2014). For myelin repair following demyelination of the mouse corpus callosum, both subtypes appear beneficial as elucidated from cell-depleting experiments where an initial proinflammatory environment promotes the proliferation of oligodendrocyte precursor cells (OPCs) whereas the later anti-inflammatory condition facilitates their development into oligodendrocytes (Miron et al., 2013; Miron and Franklin, 2014).

Given the pro-repair effects of IL4/IL13, strategies aimed at boosting the efficiency of these cytokines on macrophage functions would be highly desirable for the treatment of CNS lesions, including in demyelinating diseases. To this end, we pursued a series of experiments to test the hypothesis that stimulation with LPS, which promotes debris elimination as aforementioned, could enhance the IL4/IL13 reparative properties. Our collective results highlight that integration of pro-inflammatory LPS and regulatory IL4/IL13 axes generates a previously unappreciated, and particularly reparative, state of macrophages/microglia. Mobilizing these reparative myeloid cells pharmacologically could pave the way toward beneficial inflammation for recovery in neurologic diseases.

\section{Materials and Methods}

Bone marrow-derived macrophages (BMDMs)

Femurs from female $\mathrm{C} 57 \mathrm{Bl} / 6$ mice were isolated, and cells were flushed into a culture plate with cold DMEM supplemented with supernatant from L929 cell line enriched in macrophage-colony stimulating factor. Culture method was as previously described (Doring et al., 2015). Cells were enriched for BMDM by growing in DMEM supplemented with L929 supernatant for $7 \mathrm{~d}$, followed by culture in DMEM with 10\% FBS for a day before experiments. Medium during experiments was DMEM with $1 \%$ FBS unless otherwise specified. The following conditions were evaluated: IFN- $\gamma(10 \mathrm{ng} / \mathrm{ml})$ and LPS $(100 \mathrm{ng} / \mathrm{ml})$, IL4 $(20 \mathrm{ng} / \mathrm{ml}) / \mathrm{IL} 13$ $(10 \mathrm{ng} / \mathrm{ml}), \mathrm{LPS} / \mathrm{IL} 4 / \mathrm{IL} 13$ or vehicle (saline) for either $6 \mathrm{~h}$ for RNA or 24 $\mathrm{h}$ for protein assays. Cell-conditioned medium was removed for TNF- $\alpha$ (Invitrogen) or heparin binding epidermal growth factor (HBEGF, LifeSpan BioSciences) ELISA, or for mouse Cytokine/Chemokine 31plex array (Eve Technologies). Cell-conditioned media were concentrated 10 times to assess matrix MMP-9 by zymography (Larsen et al., 2003).

Macrophage phenotype was evaluated using ImageXpress ${ }^{\text {Micro }}$ instrumentation (IXM, Molecular Devices), a high-throughput cellular imaging system (Keough et al., 2016). Arginase assays were performed as described by Classen et al. (2009), whereas nitrite levels (as an NO indicator) were assessed by the Griess reagent system (Promega).

Cocultures of OPCs or neurons with macrophages/microglia

OPCs and microglia were purified from mixed glial cultures generated from the brains of neonatal CD-1 mice as described previously (Keough et al., 2016). Mouse cortical neurons were prepared and were plated in 96-well plates at a density of $5.0 \times 10^{4}$ cells/well; cells were left to grow for $48 \mathrm{~h}$ in B27 supplemented neurobasal media. The medium was then changed to neurobasal medium but without B27 supplement, and BMDMs were overlaid. Cells were then exposed to different treatments for $24 \mathrm{~h}$, fixed with $4 \% \mathrm{PFA}$, and were stained for microtubule associated protein-2, a neuronal marker, and the number of neurons remaining on each well was then enumerated using ImageXpress. In the case of cocultures of OPC and microglia, cells from the same mouse (10,000 OPCs: 10,000 microglia per well) were used. Cells were fixed with $4 \%$ PFA, and OPCs were identified with the $\mathrm{O} 4$ stain. The extent of process outgrowth was evaluated using ImageXpress (Keough et al., 2016).

\section{Western blot analyses}

One million BMDMs were treated for $24 \mathrm{~h}$, and cells were then lysed; 20 $\mu \mathrm{g}$ of cell extracts was separated by SDS-PAGE and transferred onto PVDF membranes that were then probed for iNOS (purified mouse anti-iNOS, BD Bioscience) or Arg-1 (Arg-1 antibody, Santa Cruz Biotechnology). For evaluating phospho-proteins, BMDMs treated for 10 or 30 min were lysed in protease inhibitor (Roche)-containing RIPA buffer (Thermo Fisher Scientific). The phospho antibodies were purchased from Cell Signaling Technology, and $\beta$-actin (Abcam) served as an internal loading control. The densitometry of immunoblots was done using ImageJ software (National Institutes of Health).

\section{Chemotaxis}

BMDMs that were treated for $24 \mathrm{~h}$ were collected and resuspended in DMEM; 200,000 cells were plated onto the filters of $5 \mu \mathrm{m}$ pore size ChemoTx plates (NeuroProbe). Recombinant murine CCL2 (PeproTech) $(10 \mathrm{ng} / \mathrm{ml})$ was added into the well below the filter so as to provide a chemotactic stimulus. Two controls were used: the first was media only while the second was a chemokinetic control where the cells plated onto the filter contained the $10 \mathrm{ng} / \mathrm{ml}$ of CCL2 as in the underlying well. To obtain a standard curve, halving numbers of cells were plated ranging from 0 to 200,000 . Cells were incubated at $37^{\circ} \mathrm{C}$ for $16 \mathrm{~h}$. They were then washed off the top of the filter and the plate spun at $1400 \mathrm{rpm}$ for $10 \mathrm{~min}$ at room temperature; $150 \mu \mathrm{l}$ of the media was discarded in the microplate and replaced with $15 \mu \mathrm{l}$ of Alamar Blue (Invitrogen). The plate was then placed at $37^{\circ} \mathrm{C}$ in humidified air with $5 \% \mathrm{CO}_{2}$ for $4 \mathrm{~h}$, and signal was read at $570 \mathrm{~nm}$.

\section{Phagocytosis}

A total of 300,000 cells/well were seeded into 12-well plates and exposed to different agents for $24 \mathrm{~h}$. The cells were further incubated with $200 \mu \mathrm{l}$ of $1 \mathrm{mg} / \mathrm{ml}$ pHrodo Red Staphylococcus aureus Bioparticles Conjugate (Thermo Fisher Scientific) for $10 \mathrm{~min}$, washed, and then harvested with 
ice-chilled PBS before acquisition by flow cytometer (BD FACS Aria II, BD Bioscience). Data were analyzed with FlowJo software.

Measurement of oxygen consumption rate (OCR) in the macrophages OCR measurements were performed using the XFe24 Extracellular Flux analyzer (Seahorse Bioscience). BMDMs were seeded at 250,000/well in XFe24 cell culture plates (Seahorse Bioscience) in $200 \mu$ l of DMEM containing $10 \% \mathrm{FBS}$. The cells were incubated overnight at $37^{\circ} \mathrm{C}$ and were then treated with test agents for $24 \mathrm{~h}$. Before performing an assay, growth medium in the wells of an XFe cell plate was exchanged with the $\mathrm{XF}$ assay medium (Agilent Technologies). While sensor cartridges were calibrated, cell plates were incubated in a $37^{\circ} \mathrm{C} /$ non- $-\mathrm{CO}_{2}$ incubator for $60 \mathrm{~min}$ before the start of an assay. All experiments were performed at $37^{\circ} \mathrm{C}$. Oligomycin (Enzo Life Sciences) (abolishes the oxygen consumption but has no effect on uncoupler-stimulated respiration), FCCP (Sigma Millipore) (p-trifluoromethoxy carbonyl cyanide phenyl hydrazine, uncoupler), and antimycin A (Sigma Millipore) (inhibit mitochondrial respiration in presence of uncoupler) were injected into the medium at final concentrations of $1,0.25$, and $0.25 \mu \mathrm{M}$, respectively. The OCR were automatically recorded and calculated by the Seahorse XFe software as per manufacturer's recommendation. The cells were lysed to estimate protein quantity by BCA (Sigma Millipore) assay in each well after the assay. The protein amounts were used to normalize OCR.

\section{HBEGF and lactate exposure to OPC growing in suboptimal media}

Murine OPCs seeded at 10,000 cells/well of 96-well plates were exposed to a suboptimal medium of DMEM without growth factor supplements. After $24 \mathrm{~h}$, cells were treated with HBEGF (Sigma Millipore; $300 \mathrm{ng} / \mathrm{ml}$ ) or lactate (Sigma Millipore; $10 \mathrm{~mm}$ ) alone or together for the next $24 \mathrm{~h}$. The cells were fixed, stained with the $\mathrm{O} 4$ and MBP antibodies, and mean $\mathrm{O}^{+}$outgrowth per cell was assessed by ImageXpress/MetaXpress (Keough et al., 2016). For rank order analysis of MBP staining (Mishra et al., 2014), a blinded examiner ranked a representative image from each sample against others, with a "winner" and "loser" decided for each pairing. Thus, for an experiment involving 16 samples, a total of $16 \times 15$ scores was obtained. The total number of "wins" (i.e., greatest amount of MBP staining) was tallied for each condition, and the highest number of "wins" was scored the highest rank, and so on.

\section{$q R T-P C R$ and microarray}

After $6 \mathrm{~h}$ of treatment, BMDMs were lysed into $1 \mathrm{ml}$ of TRIzol reagent, and RNA was extracted as per the manufacturer's instructions (Invitrogen). RNA was then reverse-transcribed using Superscript II reverse transcriptase (Invitrogen). The resulting cDNA was used as a template for the Bio-Rad iCycler MyiQ detection system and $2 \times$ SYBR Green mastermix (QIAGEN). Every primer that was used was purchased from QIAGEN, and details can be found on their homepage. Expression of gene transcripts was normalized against at least two housekeeping genes (GAPDH and $\beta$-actin). Relative expression levels for genes of interest was determined using the formula $2^{-\Delta \mathrm{CT}}$ where $\Delta \mathrm{CT}=\mathrm{CT}$ (gene of interest) - CT (housekeeping gene).

RNA quality was assessed by bioanalyzer to check the purity; then the samples were subjected to mouse gene 1.0ST array. Microarray data were analyzed with the transcriptome analysis console (Thermo Fisher Scientific).

\section{Chromatin immunoprecipitation (ChIP)}

BMDMs were first cross-linked with $1 \%$ formaldehyde in PBS (10 min incubation at room temperature). Formaldehyde was then quenched by adding glycine to a final concentration of $0.125 \mathrm{M}$ and subsequent incubation at room temperature for $5 \mathrm{~min}$. Cells were then washed, gently scrapped in PBS, spun, aliquoted $\left(1 \times 10^{6}\right)$, and snap frozen and stored at $-80^{\circ} \mathrm{C}$ until ChIP. Sodium butyrate (Sigma Millipore) at a final concentration of $1 \mathrm{~mm}$ was added to each solution.

ChIP for histone modification H3K27ac was performed as follows, using 1 million BMDM per assay. First, BMDMs were briefly thawed on ice and lysed by incubation in $1 \mathrm{ml}$ of lysis buffer (0.5\% IGEPAL CA-630, 10 mm HEPES, pH 7.9, 85 mm KCl, 1 mм EDTA, pH 8.0, in water) for $10 \mathrm{~min}$ on ice. Lysates were centrifuged at $800 \mathrm{RCF}$ for $5 \mathrm{~min}$ at $4^{\circ} \mathrm{C}$, and pellets were resuspended in $200 \mu \mathrm{l}$ of sonication/immunoprecipitation buffer (10 mм Tris-HCl, pH 7.5, 100 mм NaCl, 0.5 mм EGTA, 0.1\% deoxycholate, $0.5 \%$ sarkosyl, in water). Sonication was performed with a Bioruptor Standard Sonicator (Diagenode) and consisted of two rounds of 15 min each, alternating stages of $30 \mathrm{~s}$ "sonication-on" with $90 \mathrm{~s}$ "sonication-off"; $20 \mu$ l of $10 \%$ Triton-X was then added to samples ( $1 \%$ final concentration) on ice, and lysates were cleared by centrifugation for $5 \mathrm{~min}$ at $18,000 \mathrm{RCF}$ at $4^{\circ} \mathrm{C} ; 2 \mu \mathrm{l}$ of supernatant was then set aside for input library sequencing controls. Each sample supernatant was then immunoprecipitated on a rotator for $2 \mathrm{~h}$ at $4^{\circ} \mathrm{C}$ with $2.5 \mu \mathrm{g} \mathrm{H} 3 \mathrm{~K} 27 \mathrm{ac}$ antibody (Active Motif, mAb, 39685) prebound to $20 \mu$ l Protein A Dynabeads (Invitrogen, 10001D). Immunoprecipitates were washed with ice-cold wash buffer I (150 mu NaCl, 1\% Triton X-100, 0.1\% SDS, 2 mm EDTA pH 8.0 in water), wash buffer III (10 mm Tris-HCl, $250 \mathrm{~mm} \mathrm{LiCl,} \mathrm{1 \%} \mathrm{IGEPAL} \mathrm{CA-630,} \mathrm{0.7 \%}$ deoxycholate, $1 \mathrm{~mm}$ EDTA in water), and TET (10 mM Tris-HCl, $\mathrm{pH} 7.5$, 1 mm EDTA, pH 8.0, 0.1\% Tween-20, in water) and eluted with $1 \%$ SDS/ $\mathrm{TE}$ at room temperature in a final volume of $100 \mu \mathrm{l}$.

Reverse-crosslinking was then performed on immunoprecipitates and saved input aliquots. First, $6.38 \mu \mathrm{l}$ of $5 \mathrm{M} \mathrm{NaCl}$ (final concentration $300 \mathrm{~mm}$ ) was added to each sample immersed in $100 \mu \mathrm{l}$ SDS/TE. Crosslinking was then reversed by overnight incubation at $65^{\circ} \mathrm{C}$ in a hot air oven. Potential contaminating RNA was then digested for $1 \mathrm{~h}$ at $37^{\circ} \mathrm{C}$ with $0.33 \mathrm{mg} / \mathrm{ml} \mathrm{RNase} \mathrm{A}$, proteins were digested for $1 \mathrm{~h}$ at $55^{\circ} \mathrm{C}$ with $0.5 \mathrm{mg} / \mathrm{ml}$ proteinase $\mathrm{K}$, and DNA was extracted using Sera-Mag SpeedBeads (Thermo Fisher Scientific, 6515205050250).

\section{ChIP-seq final library preparation}

Sequencing libraries were prepared from recovered DNA (ChIP) by blunting, A-tailing, and adapter ligation as previously described (Langmead and Salzberg, 2012) using barcoded adapters (NextFlex, Bio Scientific). Libraries were PCR-amplified for 14 cycles and size-selected for fragments (200-400 bp) by gel extraction (10\% TBE gels, Invitrogen, EC62752BOX). ChIP-seq libraries were single-end sequenced for 75 cycles on an Illumina HiSeq 4000 according to the manufacturer's instruction. Samples were sequenced to obtain 15-20 million uniquely mapped reads.

\section{Data analysis for Chip-sea}

Preprocessing. FASTQ files from sequencing experiments were mapped to the mouse UCSC genome build mm10. Bowtie 2 with default parameters was used to map ChIP-seq experiments(Langmead and Salzberg, 2012). HOMER was used to convert aligned reads into "tag directories" for further analysis.

H3K27ac-enriched region calling. Homer suite of computational analyses programs were used for downstream analyses (Heinz et al., 2010). For each experiment, regions positive for H3K27ac were first called with input DNA as background using HOMER's "findPeaks" command with the "-style histone" and associated default parameters. For differential enrichment analysis, LPS/IL4/IL13 regions were first annotated with data from the LPS/IL4/IL13 and Vehicle replicates (annotatePeak. pl). We then determined regions significantly enriched in LPS/IL4/IL13 compared with Vehicle using getDiffExpression. pl. Threshold for statistical significance was as follows: twofold higher abundance at a false discovery rate of 0.05 . Regions "significantly up" in LPS/ IL4/IL13 were then compared with LPS-alone and IL4/IL13-alone conditions, using the same overall approach (annotatePeaks. pl with relevant replicates followed by getDiffExpression. $\mathrm{pl}$ and the same statistical criteria).

Motifs analysis. De novo motifs analyses were performed on genomic regions that display significantly higher abundance of H3K27ac in LPS/ IL4/IL13 conditions compared with any others. For this, we used Homer's findMotifGenome. pl command, using "-size given -ma." Background/control genomic sequences were generated by the program. Visualization of sequencing data using the UCSC genome browser was performed using Homer's makeMultiWigHub. pl program.

Proteomic analyses from BMDM lysates

Six million cells were seeded per $100 \mathrm{~mm}$ dish. Cells were activated either with IL4/IL13 or LPS/IL4/IL13 for $24 \mathrm{~h}$ and were then harvested in SDS containing buffer for processing the samples for liquid chromatography and tandem mass spectrometry (MS). Samples were alkylated with 
iodoacetamide, peptides were then labeled with isotopically heavy [40 $\mathrm{mm}{ }^{13} \mathrm{CD}_{2} \mathrm{O}+20 \mathrm{~mm} \mathrm{NaBH}{ }_{3} \mathrm{CN}$ (sodium cyanoborohydride)] or light [40 mM light formaldehyde $\left(\mathrm{CH}_{2} \mathrm{O}\right)+20 \mathrm{~mm} \mathrm{NaBH}{ }_{3} \mathrm{CN}$ ] labels. Samples were subjected to HPLC and MS at the Southern Alberta Mass Spectrometry core facility at the University of Calgary, Canada. Spectral data were matched to peptide sequences in the mouse UniProt protein database using the Andromeda algorithm as implemented in the MaxQuant software package version 1.6.0.1, at a peptide-spectrum match false discovery rate of $<0.01$. Significant outlier cutoff values were determined after $\log (2)$ transformation by boxplot-and-whiskers analysis using the BoxPlotR tool (Spitzer et al., 2014).

Analyses of metabolites extracted from cell culture conditioned media and spinal cords tissues by nuclear magnetic resonance (NMR) and gas chromatography (GC)-MS

GC-MS. A total of $50 \mu \mathrm{l}$ of each cell culture sample was used for metabolite extraction, which was performed using a modified Bligh and Dyer extraction and method (Bligh and Dyer, 1959). For metabolite derivatization, $50 \mu \mathrm{l}$ of $(20 \mathrm{mg} / \mathrm{ml})$ solution of methoxyamine-hydrochloride in pyridine was added to each tube and shaken at $37^{\circ} \mathrm{C}$ for 2.5 h. After addition of $50 \mu \mathrm{l}$ of $\mathrm{N}$-methyl-N-(trimethylsilyl) trifluoro-acetamide (Sigma Millipore) as silylating agent, the tubes were shaken at $37^{\circ} \mathrm{C}$ for another $45 \mathrm{~min}$. After dilution with $500 \mu \mathrm{l}$ of hexane and centrifugation, $200 \mu \mathrm{l}$ of supernatant was transferred to special vials for GC-MS analysis performed on a GC-TOF-MS Agilent chromatograph 7890 coupled with a Waters GCT mass spectrometers. MS range of 50-800 $\mathrm{m} / \mathrm{z}$ was used for scanning. Acquired spectra were processed using Metabolite-Detector software (version 2.1N). For the purpose of metabolite identification, an in-house library based on the GOLM metabolome database and NIST 2011 library were used. Identified peak intensities were normalized for each sample using median fold-change normalization, and missing values were imputed with the smallest value in the dataset.

NMR. A total of $200 \mu \mathrm{l}$ of each cell culture-conditioned medium was brought to $600 \mu \mathrm{l}$ by adding D2O $(60 \mu \mathrm{l}), 10 \mu \mathrm{l}$ of $1 \mathrm{M}$ sodium azide solution to prevent microbial growth and $330 \mu \mathrm{l}$ of sodium PB $(0.1 \mathrm{M})$ containing dimethyl-silapentane-sulfonate $(0.5 \mathrm{~mm})$ as chemical shift reference. The $\mathrm{pH}$ of each sample was adjusted to $7.0 \pm 0.4 .{ }^{1 \mathrm{H}} \mathrm{NMR}$ spectroscopy was performed using a modified method previously described (Weljie et al., 2007). Briefly, all experiments were performed on a Bruker Avance $600 \mathrm{NMR}$ spectrometer operating at $600.22 \mathrm{MHz}$ and equipped with a $5 \mathrm{~mm}$ TXI probe at $298 \mathrm{~K}$. One-dimensional ${ }^{1 \mathrm{H}} \mathrm{NMR}$ spectra were obtained using a standard Bruker pulse sequence program (Bruker NOSYGPRD.2). Spectra were acquired as a series of 1024 scans. After Fourier transformation, all spectra were manually corrected for phase and baseline and referenced to dimethyl-silapentanesulfonate peak at $0 \mathrm{ppm}$ using TopSpin (Bruker BioSpin) software. Metabolites were identified and quantified using the targeted profiling approach as implemented in the Chenomx NMR Suite 7.5 software (Chenomx). 2D total correlation (TOCSY) and ${ }^{1} \mathrm{H}^{13} \mathrm{C}$ heteronuclear single quantum coherence spectra, as well as the human metabolome database (version 2.5) were used to confirm the chemical shift assignments. Metabolite intensities were integrally normalized against the sum of the metabolite intensities for each sample, to adjust for possible intersample concentration variations.

Tissue extraction was performed as follows: Spinal cords were dissected, and a $3 \mathrm{~mm}$ hemisection containing the dorsal lesion was isolated; $150 \mu \mathrm{l}$ of PBS with protease inhibitor and phosphostop was added to spinal cord segment. The mixture was sonicated on ice 3 times for $5 \mathrm{~s}$ each using a sonic dismembrator. The metabolites were extracted in organic solvents.

Data processing and statistical analysis of metabolites. Preprocessed GC-MS and NMR data were log-transformed, centered, and unit-variance scaled within the SIMCA multivariate analytical software (version 14.0.0, Umetrics) before generating the models. A preliminary principal component analysis was generated for each comparison to identify the potential variables that could form distinct sample subsets, as well as to detect potential outlier samples. The quality of the multivariate models was evaluated by $R^{2} Y$ and $Q^{2} Y$ scores based on a sevenfold internal cross-validation routine. The $\mathrm{R}^{2} \mathrm{Y}$ shows the percentage of variation described by the model, while $\mathrm{Q}^{2} \mathrm{Y}$ is indicative of the predictive ability of the model. The model's goodness of fit is described by the difference of these two values (Picard and Cook, 1984). The choice of input variables for partial least squares determinant analysis (OPLS-DA) analysis was based on the magnitude of the variable influence on projection. Metabolites with variable influence on projection of less than a defined threshold were further filtered. For each analysis, the variable influence on projection threshold was set so that $\mathrm{R}^{2} \mathrm{Y}$ and $\mathrm{Q}^{2} \mathrm{Y}$ were highest, and their difference was minimal. To inspect the validity and potential over fit in the PLS-based models, a 999 times permutation test was used for the outcomes of interest. Results are presented as $Q^{2}$ intercepts. A $Q^{2}$ intercept of zero or negative demonstrates the stability and nonrandomness of the model, and therefore strongly supports the validity of the model.

\section{Lysolecithin demyelination}

All animal procedures were approved by the local ethics committee in accordance with guidelines of the Canadian Council of Animal Care. For live imaging experiments, CX3CR1 ${ }^{\mathrm{GFP} /+}$ (RRID:IMSR_JAX:005582) mice were crossed with Thy1 ${ }^{\text {YFP+ }}$ (RRID:IMSR_JAX:003782) mice to visualize both microglia/macrophage and axons concurrently (Stirling et al., 2014). To attempt to delineate microglia from monocyte-derived macrophages in the CNS, homozygous CCR $2^{\mathrm{RFP} / \mathrm{RFP}}: \mathrm{CX} 3 \mathrm{CR} 1^{\mathrm{GFP} / \mathrm{GFP}}$ mice (obtained from Paul Kubes, University of Calgary) were crossed with $\mathrm{C} 57 \mathrm{Bl} / 6$ mice (Charles River Laboratories) to generate CCR2 ${ }^{\mathrm{RFP} /+}$ : $\mathrm{CX} 3 \mathrm{CR} 1^{\mathrm{GFP} /+}$ mice. However, while the initial intention was to ascribe microglia as $\mathrm{CX} 3 \mathrm{CR} 1^{+}$and monocyte-derived macrophages as $\mathrm{CCR} 2^{+}$, monocytes in the periphery can express both CX3CR 1 and CCR2, whereas microglia in the CNS may express CCR2 (Burgess et al., 2019; Komiya et al., 2020). Thus, we transitioned to CX3CR $1^{\mathrm{CreER} /{ }^{+}}$: Rosa26 $^{\text {tdTom }}$ mice. CX3CR1 ${ }^{\text {CreER }}$ (JAX 021160) and Rosa26 $6^{\text {tdTom }}$ (tdTomato; JAX 007905) male and female mice on the C57BL/6 background were obtained from The Jackson Laboratory and crossed at the University of Calgary animal facility to generate CX3CR $1^{\mathrm{CreER} /+}$ : Rosa $26^{\text {tdTom } /+}$ mice. Pups received their tamoxifen at $\sim 2$ weeks of age; this comprised three intraperitoneal doses of $1 \mathrm{mg}$ tamoxifen with a separation of $24 \mathrm{~h}$ between doses. Microglia, being self-renewing, are permanently labeled with tdTomato after tamoxifen treatment, whereas monocytes (and hence macrophages when they invaded tissues) are regularly generated from bone marrow stem cells and are negative for tdTomato label by 4 weeks after tamoxifen (Plemel et al., 2020).

We also purchased C57BL/6 mice (Charles River Laboratories) for all other experiments. Female mice between 8 and 10 weeks of age were anesthetized with a mixture of ketamine $(100 \mathrm{mg} / \mathrm{kg})$ and xylazine $(10 \mathrm{mg} / \mathrm{kg})$. The analgesic buprenorphine $(0.05 \mathrm{mg} / \mathrm{kg})$ was administered subcutaneously immediately before and $12-16 \mathrm{~h}$ after surgery. The dorsal spinal cord was exposed between the T3 and T4 thoracic vertebra using spring scissors, and a 30 gauge metal needle was used to clear the dura. We used a pulled glass microcapillary attached to a $10 \mu \mathrm{l}$ syringe to deposit $1.0 \mu \mathrm{l}$ volume of $1 \% \mathrm{D}$-lysophosphatidylcholine (lysolecithin; Sigma Millipore) solution over $2 \mathrm{~min}$ in the dorsal column. At day 3 , sutures were reopened to locally deposit the test mixtures: saline, IFN- $\gamma$ (33.3 ng)/LPS (166.5 $\mu \mathrm{g})$, IL4 (66.6 ng)/IL13 (33.3 ng), and LPS/IL4/IL13. These doses were based on previously published work (Lee et al., 2010; Fenn et al., 2014). Animals were killed at $5 \mathrm{~d}$ after demyelination (i.e., $2 \mathrm{~d}$ after cytokine administration) for multiphoton ex vivo imaging as described previously (Plemel et al., 2018) at $7 \mathrm{~d}$ for OPC and oligodendrocyte enumeration, or at $21 \mathrm{~d}$ for electron microscopy.

We note that, while there are reports of minimal loss of axons and complete remyelination following lysolecithin demyelination of the spinal cord (Huang et al., 2012), others describe a substantial attrition of axons after the same insult (El Waly et al., 2020). Our own experience with dorsal column demyelination with lysolecithin is substantial axonal degeneration with incomplete remyelination at later time points (Skihar et al., 2009; Lau et al., 2012).

With regards to tissue harvest for immunohistochemistry (d 7), animals were transcardially perfused with $20 \mathrm{ml}$ room temperature PBS 
followed by $20 \mathrm{ml}$ ice-cold 4\% PFA in PBS. The spinal cords were isolated and postfixed in $4 \%$ PFA overnight at $4^{\circ} \mathrm{C}$, cryoprotected with $30 \%$ sucrose at $4^{\circ} \mathrm{C}$ for $72 \mathrm{~h}$, cut into $6 \mathrm{~mm}$ pieces with the injection site in the center, and frozen in OCT compound in moulds (up to five cords per mold) resting on a mixture of dry ice and 2-methyl-butane. The blocks were stored at $-80^{\circ} \mathrm{C}$ until ready to section. Here, the cords were cut into $20 \mu \mathrm{m}$ coronal sections over a series of 10 sets of slides, such that adjacent sections on a single slide were $200 \mu \mathrm{m}$ apart. The slides were kept at $-20^{\circ} \mathrm{C}$ until ready to process for histology.

\section{Staining of tissue sections}

To assess the lesion size at epicenter, one series of coronal spinal cord sections was stained for myelin with the eriochrome cyanine protocol as previously described (Keough et al., 2016). To evaluate the extent of demyelination in ericohrome cyanine-stained sections, we used the ImageJ program (National Institutes of Health) to measure the area within each dorsal column that did not contain blue staining. We used grid tool in the ImageJ program (National Institutes of Health) to assess the lesion volume across the group.

For immunofluorescence staining, sections were blocked for $30 \mathrm{~min}$ with serum-containing buffer, incubated at $4^{\circ} \mathrm{C}$ overnight with primary antibodies followed by $1 \mathrm{~h}$ at room temperature with secondary antibodies. For staining of versican core protein, chondroitinase $A B C(0.2 \mathrm{U} / \mathrm{ml}$ in PBS) was added for $30 \mathrm{~min}$ at $37^{\circ} \mathrm{C}$ to remove glycosaminoglycan side chains, followed by a PBS rinse. The sections were postfixed in methanol at $-20^{\circ} \mathrm{C}$ for $10 \mathrm{~min}$, rinsed with PBS, and blocked with $10 \%$ horse serum containing $1 \%$ BSA and $0.1 \%$ cold fish-skin gelatin. Then primary and secondary antibodies were used as above. The following primary antibodies were used: anti-Iba-1 (1:500, macrophage/microglia, Wako Chemicals), anti-iNOS (1:50, BD Bioscience), anti-CD204 (1:50, BioRad), anti-Arg-1 (1:50, Cell Signaling), anti-platelet-derived growth factor $\alpha$ receptor (1:50, PDGFR $\alpha$, for OPCs), anti-APC (1:50, adenomatus polyposis coli (CC1); for oligodendrocytes, Calbiochem), and (1:100) anti-versican gag $\beta$ (versican V1). AlexaFluor-488 (1:750, Invitrogen) or AlexaFluor-564 (1:750, Invitrogen) secondary antibodies were used to visualize the primary antibodies. Images $(\times 10$ and $\times 60)$ were taken on an Olympus FluoView FV10i confocal microscope. Initial experiments to detect Arg-1 in vivo were unsuccessful so CD204 (macrophage scavenger receptor that stains regulatory and phagocytic cells) was the focus; later experiments were successful for Arg-1 labeling with a new batch of antibody and thus Arg-1 was incorporated into later experiments.

For ex vivo real-time imaging, at day 5 following injury $(2 \mathrm{~d}$ after the local application of test solutions), CX3CR1 ${ }^{\mathrm{GFP}} / \mathrm{Thy} 1^{\mathrm{YFP}}$ mice were killed and the dissected spinal cord was suspended in an ACSF perfusion chamber where they remain viable for at least $4 \mathrm{~h}$ (Stirling et al., 2014; Plemel et al., 2018). Myelin was visualized by the lipophilic dye Nile Red added to the perfusion chamber. Live imaging over $2 \mathrm{~h}$ was performed with a multiphoton spectral microscope, and postacquisition analyses using advanced optical imaging software ImageTrak (Jensen et al., 2018) (Peter Stys, University of Calgary) enabled spectral unmixing. The surface rendering and measurements of the motility of individual CX3CR1GFP macrophages/microglia were done by IMARIS software (Bitplane).

Toluidine blue-stained semithin sections and g-ratio analyses

This was performed as previously described (Keough et al., 2016). At least 200 axons were evaluated, and the mean g-ratio per condition was then obtained. For electron microscopy, $70 \mathrm{~nm}$ sections were cut on an ultramicrotome and contrasted with lead citrate. Specimens were examined with a Hitachi 7650 electron microscope.

\section{Statistical analysis}

In the case of multiple experimental groups, one-way or two-way ANOVA test with appropriate post hoc test was used and mentioned in the figure legends. For in vitro experiments, the $n$ indicates the number of cultures (normally $n=3$ or 4 cultures) per group and where the data were reproduced in three different experiments. The ChIP sequencing experiment from activated BMDMs $n=3$ per condition was performed once. For the in vivo data, the $n$ indicates the number of mice where we have used 4-6 mice/condition, and where manipulations were repeated in at least two different experiments. For ex vivo analyses, 3 or 4 mice per group were analyzed. For cell density measurements, each mouse was considered an $n$ of 1 . For morphology and motility measurements in demyelinated lesions in vehicle and LPS/IL4/IL13-treated mice, each cell was considered an $n$ of 1 , and between 38 and 145 cells were quantified per mouse across 3 or 4 mice per group. For analysis of the percentage of phagocytosis in ex vivo samples, the colocalization of Nile red at each time point was considered an $n$ of 1 , and 7 time points were assessed per mouse across 3 or 4 mice.

\section{Results}

\section{Distinct characteristics of M(LPS/IL4/IL13) macrophages} (M) or Mi(LPS/IL4/IL13) microglia (Mi) in culture

We generated BMDMs of high purity from precursors differentiated through macrophage-colony stimulatory factor treatment (Doring et al., 2015). In turn, we polarized cells toward proinflammatory and regulatory states using IFN- $\gamma$ and LPS (IFN/ LPS) and IL4/IL13, respectively. We found that macrophages treated with IFN/LPS elevated pro-inflammatory cytokines (e.g., IL12) (Fig. 1a), chemokines (e.g., CXCL3) (Extended Data Fig. 1-1), and transcripts [Inos (inducible nitric oxide synthase), Il12, and Ubiquitin C] (Extended Data Fig. 1-2). Conversely, macrophages exposed to IL4/IL13 increased expression of Arg-1 (Arg1), Ym1, and Fizz-1 (Extended Data Fig. 1-2), which is indicative of an immune-regulatory phenotype.

Strikingly, the simultaneous exposure of macrophages to IL4/ IL13 and LPS yielded features distinct from those of M(IFN/LPS) or M(IL4/IL13) characteristics. Arg-1 expression and activity in M(LPS/IL4/IL13) cells were markedly elevated over that of M (IL4/IL13) alone, while iNOS and nitrite content was either absent or marginally elevated in M(LPS/IL4/IL13) despite the presence of the LPS trigger (Fig. $1 b-d$ ). Staining of CD204 ${ }^{+}$cells indicated that the majority, rather than a subpopulation, were altered in their phenotype (Fig. 1e). Moreover, the M(LPS/IL4/ IL13) macrophages had a prominent rise of the IL10 regulatory cytokine and CCL2 chemokine, while IL12 was not detected despite the use of LPS (Fig. 1a). IL4 receptor- $\alpha$ transcript level was high in M(LPS/IL4/IL13) macrophages (Extended Data Fig. 1-2), suggesting that the combination triggers more receptors available for IL4 stimulation. Other molecules elevated by LPS/IL4/IL13 in primary macrophages (Extended Data Fig. 1-1) or microglia (Extended Data Fig. 1-3) included granulocyte-colony stimulatory factor and a number of chemokines (CCL2, CCL3, CCL4, CXCL3, and CXCL10). The latter suggests that the M(LPS/IL4/ IL13) macrophages or Mi(LPS/IL4/IL13) microglia participate in migratory behavior and support the observation that these cells are highly motile to chemotaxis by CCL2 (Fig. 1f). Finally, the M (LPS/IL4/IL13) macrophages are highly phagocytic in a pHrodo phagocytosis assay (Fig. 1g).

Pro-inflammatory and highly activated macrophages have cytotoxic properties (Laskin and Pendino, 1995), and excessive LPS stimulation has the capacity to injure CNS cells, including in coculture of oligodendrocytes and macrophages and microglia (Fabian and Rea, 1993; Li et al., 2008). Thus, we ascertained whether the inclusion of LPS in generating M(LPS/IL4/IL13) would kill neurons or oligodendrocytes in coculture with macrophages. We observed significant reduction of $\mathrm{MAP}^{+}$neurons (Fig. $1 h, j$, top), reflecting cell death with dead cells lifting off (Mishra et al., 2014), when neurons were in coculture with M(IFN/LPS) macrophages; OPCs were similarly reduced. Importantly, these neural cells did not die in M(LPS/IL4/IL13) condition. Similarly, for microglia in coculture, Mi(IFN/LPS) 
a

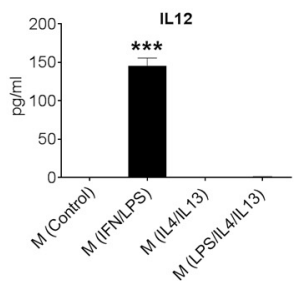

C

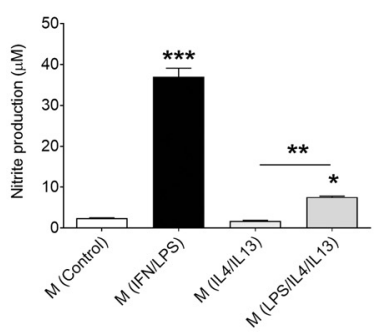

d

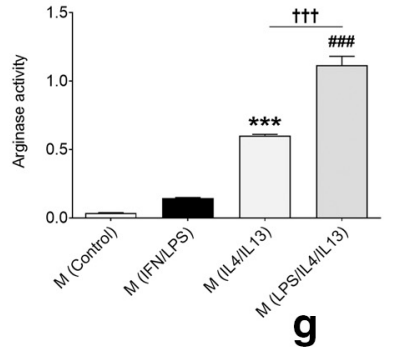

f
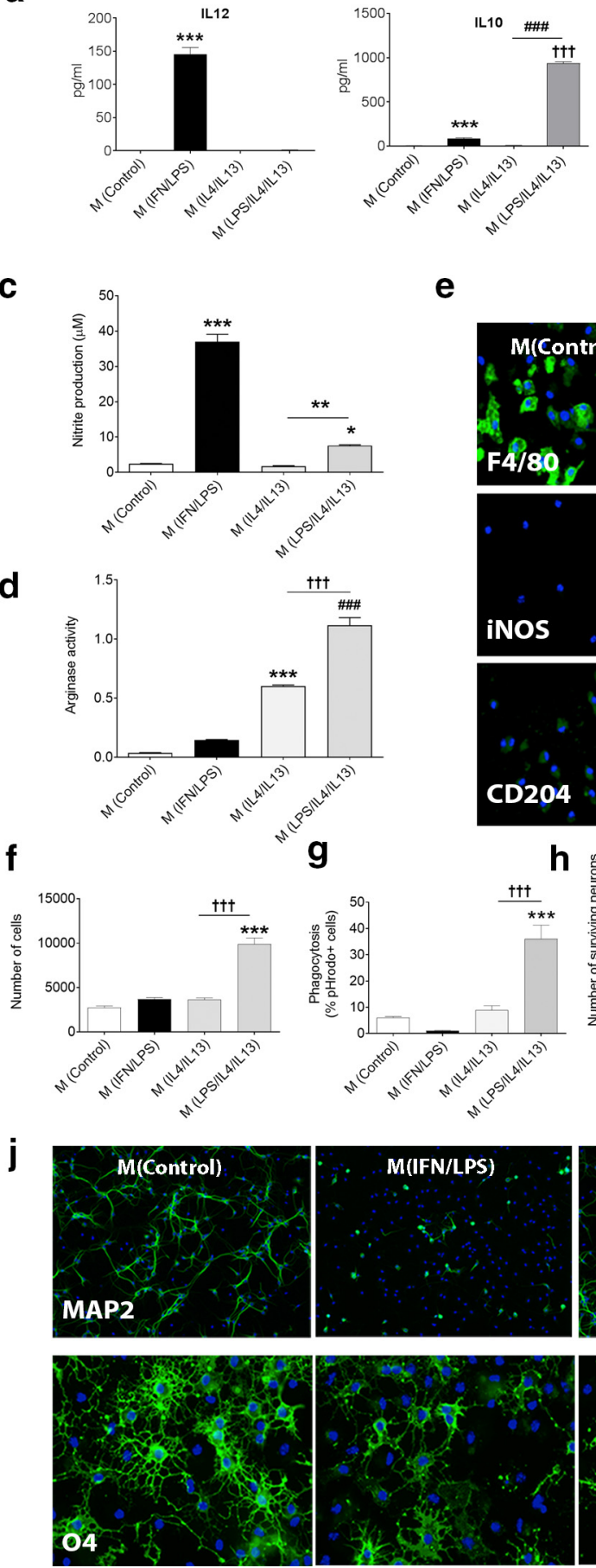

e

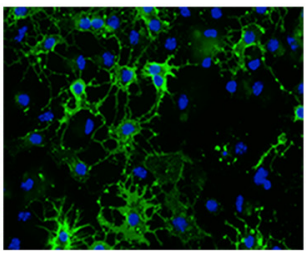

b

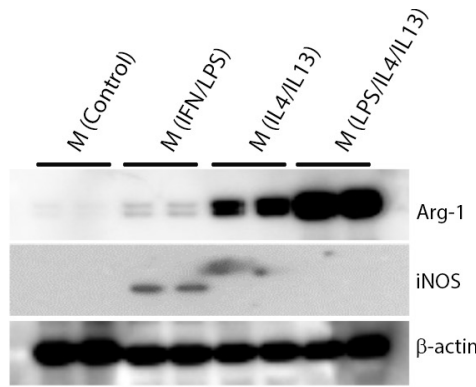

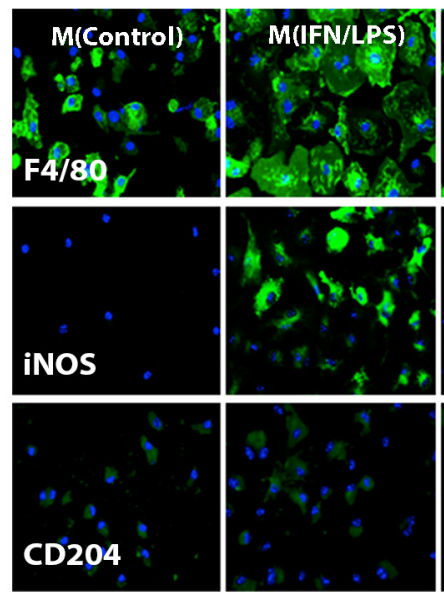

h
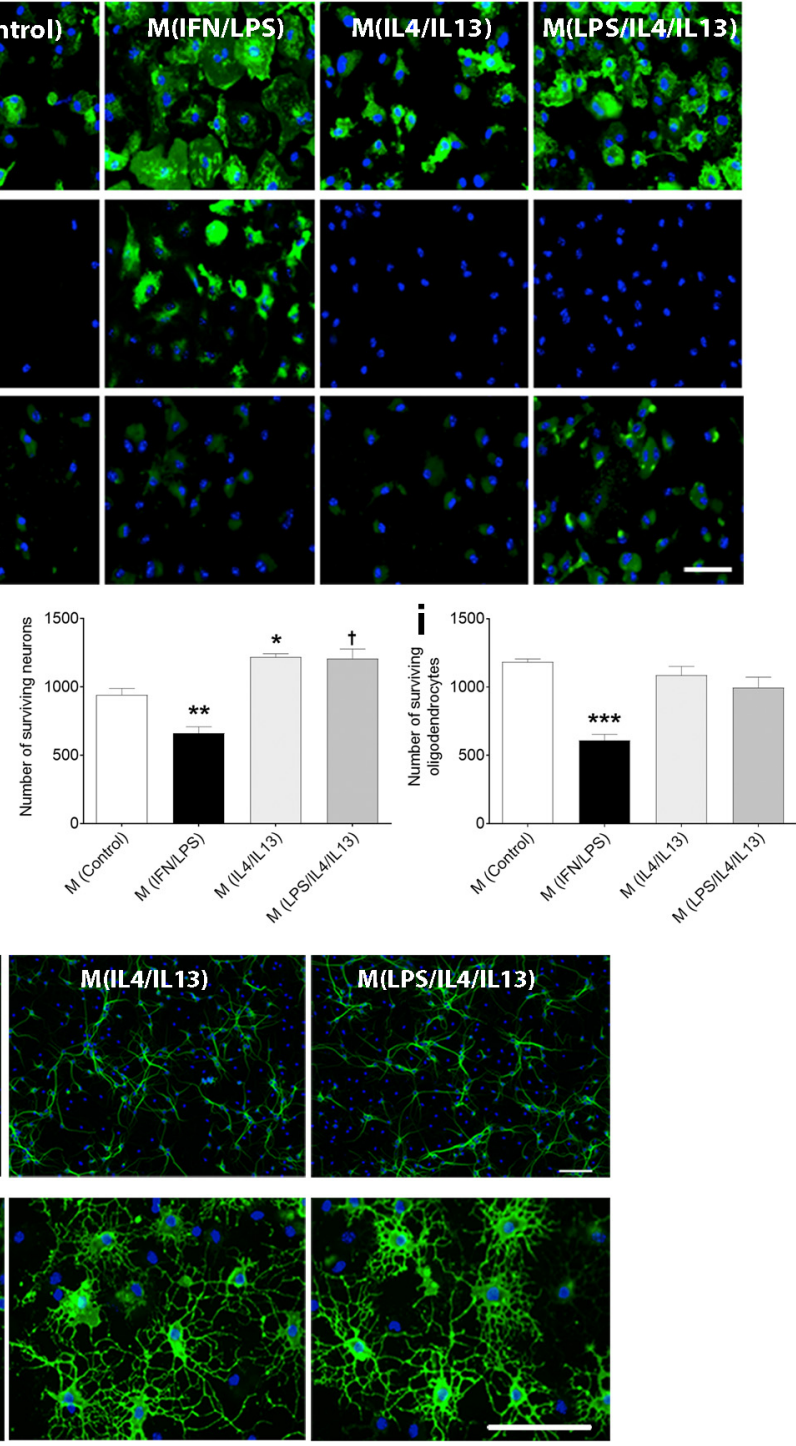

Figure 1. Features of M(LPS/IL4/L13) macrophages. $\boldsymbol{a}$, Mouse M(LPS/IL4/IL13) macrophages after $24 \mathrm{~h}$ did not secrete detectable ELISA levels of IL12 ( $n=4 ; F_{(3,11)}=2.92 ;{ }^{* * *} p=0.0001$; one-way ANOVA) and had substantially elevated IL10 $\left(n=4 ; F_{(3,12)}=2303 ;{ }^{* * *} p<0.0001 ;{ }^{\# \# \#}<<0.0001 ;+t+p<0.0001\right.$; one-way ANOVA, post hoc Tukey). Alterations of other cytokines and chemokines in M(LPS/L4/L13) are displayed in Extended Data Figure 1-1, as are transcripts encoding markers of regulatory cells (Extended Data Figure 1-2). Microglia (Mi) are similarly altered by the LPS/IL4/IL13 mixture (Extended Data Figure 1-3). $\boldsymbol{b}, \mathrm{M}(\mathrm{LPS} / \mathrm{LL} 4 / \mathrm{LL13})$ at $24 \mathrm{~h}$ had higher Arg-1 level (Western blot) than M(IL4/IL13), whereas iNOS was not induced. c, Nitrite production was highest in M(IFN/LPS) $\left(n=4 ; F_{(3,8)}=249.9 ;{ }^{* * *} p=0.0001 ;{ }^{*} p=0.0212\right.$ compared with control; ${ }^{* *} p=0.0094$; one-way ANOVA, post hoc Tukey). $\boldsymbol{d}$, Arg-1 enzyme activity was most elevated by M(LPS/LL/LL13); $n=4 ; F_{(3,8)}=228.1 ;{ }^{* * *} p=0.0001 ; \# \#=0.0001$ compared with control; $+t+p=0.0001$; one-way ANOVA, post hoc Tukey). $e$, Immunostaining showed upregulation of iNOS and CD204 in all F4/80-positive BMDM after $24 \mathrm{~h}$ in M(IFN/LPS) and M(LPS/LL/IL13) conditions, respectively. Scale bar, $100 \mu \mathrm{m} . f$, M(LPS/L4//L13) macrophages were highly motile in a chemotaxis assay $\left(n=4 ; F_{(3,12)}=73.7 ;{ }^{* * *} p=0.0001 ;+t+p=0.0001\right.$; one-way ANOVA, post hoc Tukey) and very phagocytic $(\boldsymbol{g})\left(n=3 ; F_{(3,8)}=32 ;{ }^{* * *} p=0.0003 ; \dagger+t p=0.0006\right.$; one-way ANOVA, post hoc Tukey). $\boldsymbol{h}-\boldsymbol{j}$, Cocultures of mouse neurons (microtubule-associated protein-2 [MAP2]) and BMDM ( $\boldsymbol{h}$, and top panels in $\boldsymbol{j}$ ), or microglia and OPCs (i, bottom panels in $\boldsymbol{j}$, 04 staining) were exposed to the respective stimuli for $24 \mathrm{~h}$, and toxicity was measured by the number of remaining neurons $\left(\boldsymbol{h} ; n=4 ; F_{(3,12)}=27.01 ;{ }^{*} p=0.0109 ;{ }^{* *} p=0.0094\right.$; $t p=0.0144$; one-way ANOVA, post hoc Tukey), or (i) oligodendrocytes $\left(n=4 ; F_{(3,8)}=20.13 ;{ }^{* * *} p=0.0004\right.$; one-way ANOVA, post hoc Tukey). Scale bar, $100 \mu \mathrm{m}$. All histograms are mean \pm SEM and reproduced in at least another experiment. 
had prominent toxicity to $\mathrm{O}^{+}$OPCs, whereas $\mathrm{Mi}(\mathrm{LPS} / \mathrm{IL} 4 / \mathrm{IL} 13)$ did not kill these cells (Fig. 1i,j, bottom panels).

Collectively, our results reveal a previously unknown state of macrophages/ microglia that is generated by the integration of pro-(LPS) and anti-(IL4/IL13) inflammatory triggers. The M(LPS/IL4/ IL13) or Mi(LPS/IL4/IL13) cells have antiinflammatory, migratory, and phagocytic properties beyond that of the commonly studied IL4/IL13-exposed macrophages or microglia; these cells also do not kill neurons or oligodendrocytes despite the presence of LPS in the activating condition.

As BMDMs were more readily obtained in large quantities compared with brainderived microglia, subsequent experiments in culture focused on macrophages unless otherwise stated. We evaluated the capacity of other TLR ligands in generating the M (LPS/IL4/IL13) spectrum. Figure 2 denotes that both LPS and the TLR2 agonist (PAM2) promoted arginase activity in IL4/ IL13-treated macrophages, whereas the TLR3 agonist, polyIC, was ineffective. Moreover, once generated, the M(LPS/IL4/ IL13) cells were relatively stable compared with other subsets, as demonstrated by high arginase activity over a $4 \mathrm{~d}$ period (Fig. 2b). The IL4/IL13 signal was overriding in that even when LPS was added 6 or $12 \mathrm{~h}$ before or after the IL4/IL13 treatment, the result $48 \mathrm{~h}$ after IL4/IL13 initiation was that arginase activity remained high (Fig. $2 c$ ). The increase in arginase activity in $\mathrm{M}$ (LPS/IL4/IL13) is mediated by engagement with the TLR4 axis (Fig. 2d). We found that the addition of IFN $\gamma$ to M(LPS/IL4/ IL13) macrophages caused cells to acquire features similar to those of M(IFN/LPS) (Extended Data Fig. 1-2).

\section{Signaling pathways that generate the M(LPS/IL4/IL13) phenotype}

We sought to understand how the M(LPS/IL4/IL13) phenotype was generated. First, we subjected macrophages $6 \mathrm{~h}$ after treatment to mRNA microarray analyses (NCBI GEO number GSE138263). Figure $3 a$ and Extended Data Figure 3-1a, $b$ show that there are transcriptomic changes selective to the M(LPS/IL4/ IL13) cells compared with the other groups. Pathway analyses of the microarray data (transcriptome analysis console software, Thermo Fisher Scientific), of altered transcripts in the M(LPS/ IL4/IL13) macrophages, compared with cells in all other treatment groups and control, identified a central role for the cAMPresponse element binding protein 1 (CREB1) in generating $M$ (LPS/IL4/IL13) cells (Fig. 3b). In support, the phosphorylation of CREB was highly upregulated in M(LPS/IL4/IL13) but also M (IFN/LPS) conditions (Fig. 3c). Moreover, the CREB inhibitor RMM46 decreased arginase activity of M(LPS/IL4/IL13) macrophages (Fig. 3d).

Many upstream kinases stimulate CREB1 activity. To investigate which kinase(s) influences the generation of M(LPS/IL4/ b

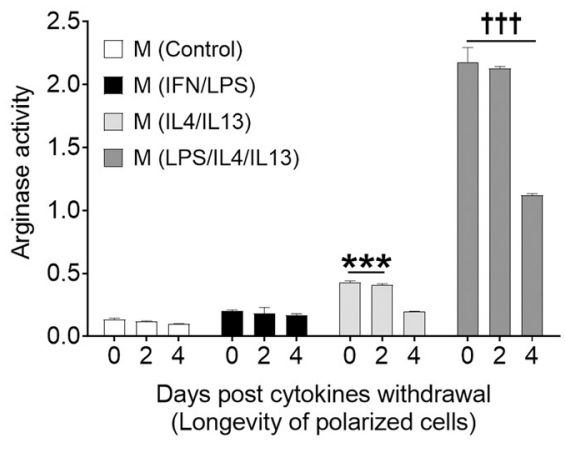

d

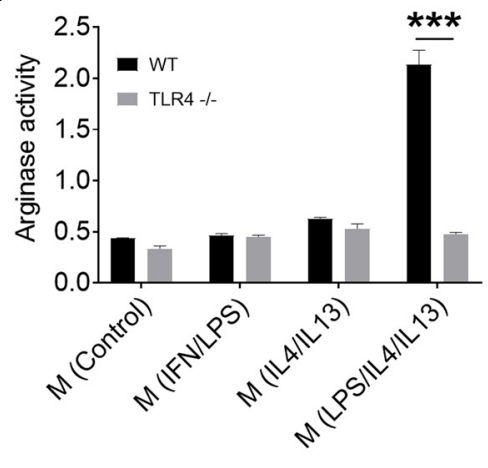

Figure 2. Macrophages were treated with TLR agonists in the presence or absence of IL4/IL13; then arginase activity was $p=0.0001 ; \quad p=0.0001 ; \dagger+t p=0.0001 ;$ one-way ANOVA). assessed the longevity of M(LPS/IL4/IL13) cells $(\boldsymbol{b})$. These cells lived longer and generated high level of arginase activity we compared the arginase activity in WT and TLR4 ${ }^{-1-}$ mice $(\boldsymbol{d})$. M(LPS/IL4/IL13) response was abrogated in TLR4 ${ }^{-l-}$ mice ( $n=3 ;{ }^{* * *} p=0.0001$; two-way ANOVA, Sidak's multiple comparison).

IL13) cells, macrophages were exposed to LPS/IL4/IL13 and subjected to mass spectroscopic analyses. We found many upstream signaling pathways elevated, such as phosphatidylinositol-3 kinase (PI3K), MAP kinases, phospholipase, and molecules related to ras signaling pathway (Fig. 3e) in M(LPS/IL4/ IL13). We also corroborated these findings in the presence of pharmacological inhibitors to signaling molecules. We found that the arginase activity elevated in M(LPS/IL4/IL13) cells was abrogated by inhibitors to PI3K (Ly294002), ERK1/2 (U0126), p38MAP kinase (SB203580), or mTOR (rapamycin) but not to an inhibitor of MMPs, BB94 (Fig. 3f). Thus, MAP kinases and PI3K/mTOR are involved in generating M(LPS/ IL4/IL13) macrophages.

Next, we investigated the upstream adaptor molecules (MyD88 and TRIF) involved in the LPS activation of the toll-like receptor-4 (TLR-4) pathway. We used BMDMs from mice genetically deficient for MyD88 or TRIF and found that arginase activity was no longer elicited by LPS/IL4/IL13 in MyD88 null compared with WT cells (Fig. $3 g$ ). The generation of M(LPS/IL4/ IL13) did not require TRIF as arginase activity remained high in TRIF $^{-1-}$ macrophages. The requirement of TLR4 in generating the M(LPS/IL4/IL13) phenotype was noted above as arginase activity was no longer promoted in TLR $4^{-1-}$ mice (Fig. $2 d$ ). 


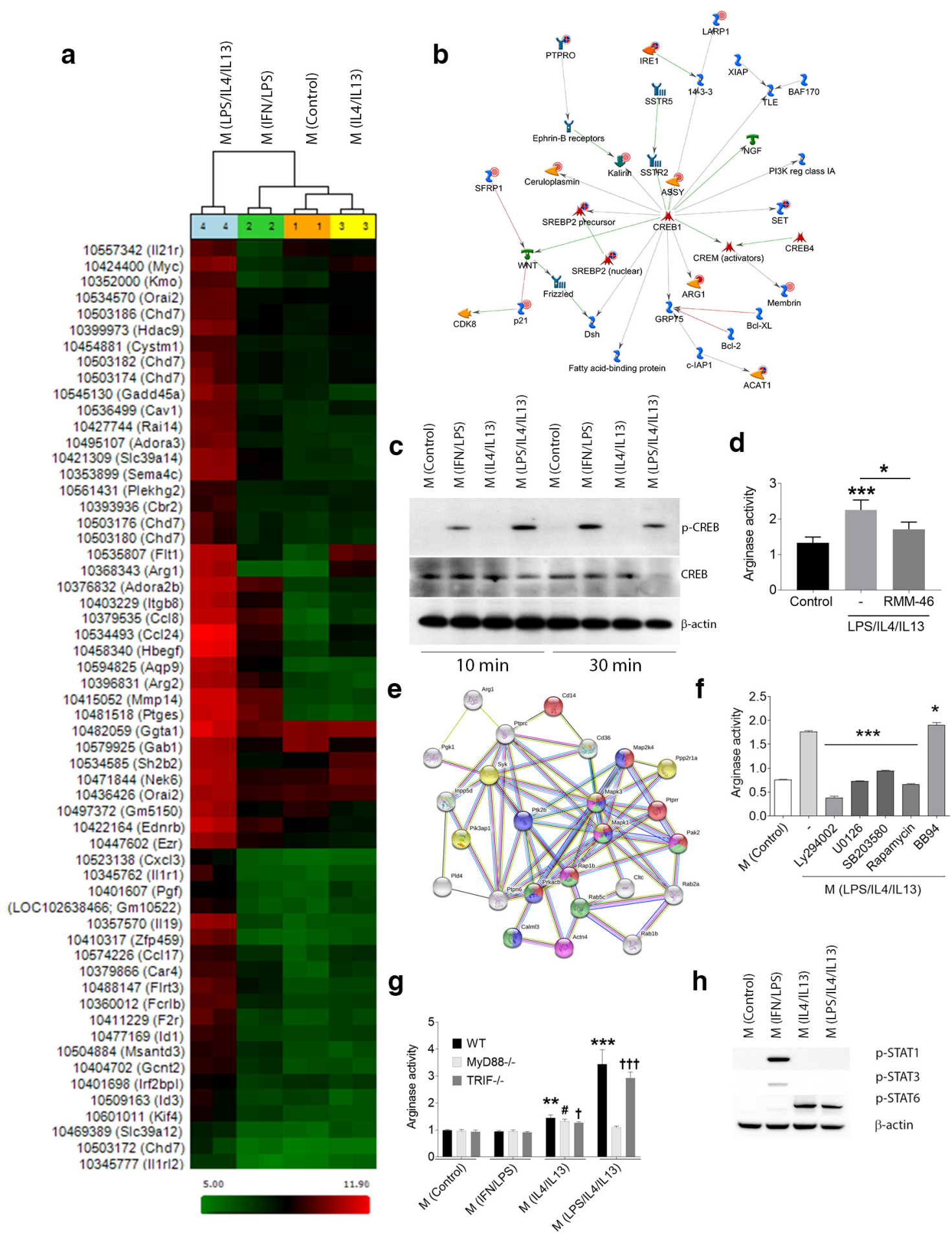

Figure 3. Signaling pathways that generate the M(LPS/L4/L13) phenotype. Differential expression of genes (log2 fold change) in macrophages revealed by (a) hierarchical clustering (GSE138263) between treatments (6h exposure, $n=2$ ). Levels of particular transcripts are displayed in Extended Data Figure 3-1. $\boldsymbol{b}$, Pathway analyses highlighted the central role of CREB signaling in generation of the M(LPS/L4/L13) phenotype. c, Immunoblot confirmed the elevated phosphorylation of CREB protein in BMDM after 10 or 30 min exposure to IFN/LPS or LPS/L4/ IL13. $\boldsymbol{d}$, Inhibiting CREB signaling reduced Arg-1 enzyme activity in M(LPS/L4/LL13) $\left(n=4 ; F_{(2,9)}=17.52 ;{ }^{*} p=0.0180 ;{ }^{* * *} p=0.0006\right.$; one-way ANOVA, post hoc Tukey). $\boldsymbol{e}$, KEGG pathway analysis displayed the prominent signaling pathways from proteomics of $M(L P S / L 4 / L 13)$. $f$, Inhibition of upstream kinases that can influence activation of CREB or STAT signaling prevented the elevation of Arg-1 enzyme activity in M(LPS/L4/IL13) $\left(\boldsymbol{g} ; n=4 ; F_{(6,14)}=513.8 ;{ }^{*} p=0.0268 ;{ }^{* * *} p=0.0001\right.$ compared with M(LPS/IL4/IL13; one-way ANOVA, post hoc Tukey). $\boldsymbol{g}$, The importance for MyD88 pathway in generating M(LPS/L4/LL13), as the elevation of Arg-1 enzyme activity after stimulation was lost in MyD88 ${ }^{-1-}$ but not TRIF ${ }^{-1-}$ BMDM $\left(n=4\right.$; ${ }^{* *} p=0.0042$; ${ }^{* * *} p=0.0001 ;{ }^{*} p=0.0308 ; t p=0.0463 ;+t+p=0.0001$; two-way ANOVA). $\boldsymbol{h}$, Representative immunoblot demonstrated the differential activation profile of phosphorylated STATs, where STAT1 and 3 were elevated in M(IFN/LPS), whereas STAT6 was activated in either M(IL4/IL13) or M(LPS/L4/LL13). 
a Polarization-independent locus

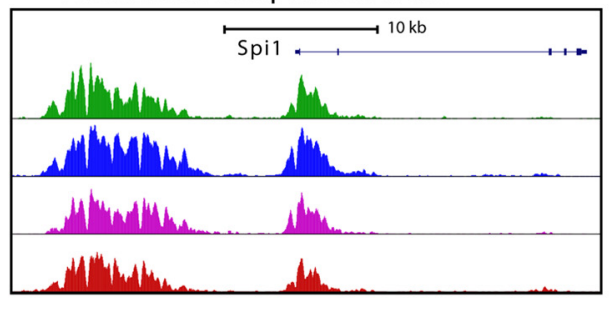

\section{LPS-responsive}

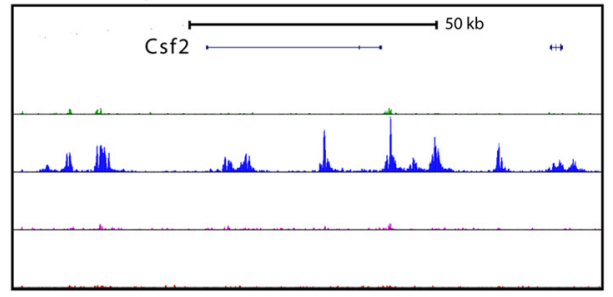

e LPS/IL4/IL13-responsive

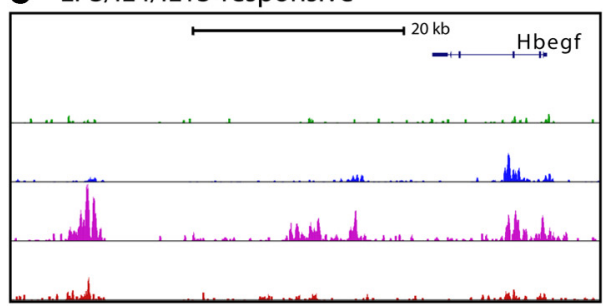

b Immune ligand-responsive

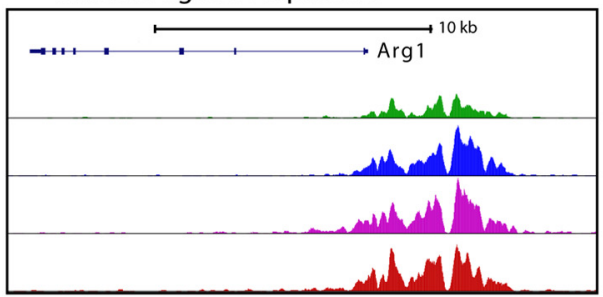

d IL4/IL13-responsive

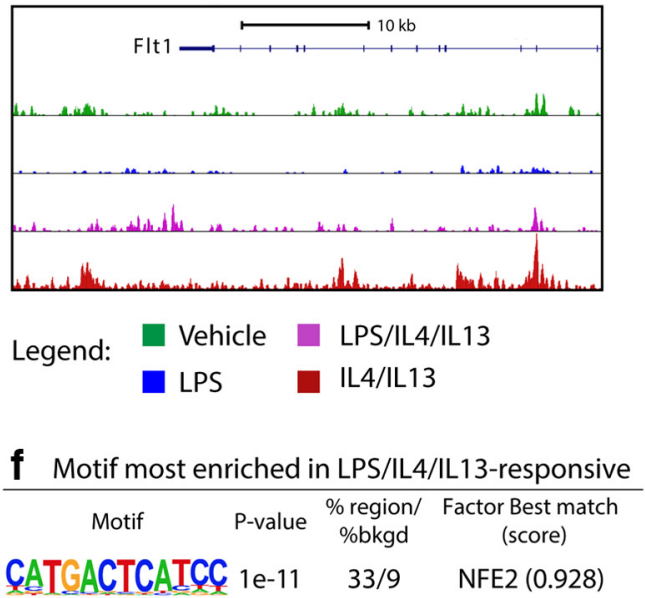

Figure 4. $\boldsymbol{a}-\boldsymbol{e}$, ChIP-seq analyses reveal stimulus-dependent gain of H3K27 acetylation at genomic regions. UCSC data visualization depicting acetylation levels at regions that are (a) insensitive to macrophage polarization (i.e., control region), (b) responsive to macrophage stimulations, (c) responsive to LPS, (d) responsive to IL4/LL13, and (e) responsive to LPS/LL/LL13. $\boldsymbol{f}$, Dominant DNA motifs enriched at regions responsive to LPS/LL/IL13 compared with other polarization mode.

The STATs (signal transducer and activator of transcription) family of transcription factors are frequently engaged following activation of macrophages. We noted that M(IFN/LPS) had activation of STAT1 and STAT3, whereas M(IL4/IL13) favored STAT6 phosphorylation. Interestingly, M(LPS/IL4/IL13) did not engage STAT1/STAT3, whereas STAT6 was activated (Fig. $3 h$ ).

To further address the signaling axis implicated, we interrogated epigenetic activity at regulatory elements on a genomewide scale by performing ChIP followed by massively parallel sequencing (i.e., ChIP-seq) experiments. For this, we targeted histone post-translational modification of acetylation at lysine 27 of histone 3 (i.e., H3K27ac) as gain/high abundance of this epigenetic mark correlates with local interactions with factors and complexes that promote transcription. Overall, each immune stimulation regimen led to significant increases in H3K27ac deposition at both stimulation-common and stimulation-specific regulatory elements throughout the genome (Fig. $4 a-e$ ). Notably, we found evidence that 122 regions display a significant gain of H3K27ac following LPS/IL4/IL13 costimulation compared with either LPS- or IL4/IL13-only stimulation (twofold higher abundance of $\mathrm{H} 3 \mathrm{~K} 27 \mathrm{ac}$ at a false discovery rate of $5 \%$ ). Furthermore, motif enrichment analyses revealed that NFE2 transcription factors might play a key role in driving activity at these LPS/IL4/ IL13-responsive sites (Fig. 4f). Therefore, these results indicate that costimulation with LPS and IL4/IL13 leads to a unique profile of signaling pathways activity, which in turn enables the distinct transcriptional program and cell behavior of LPS/IL4/IL13 macrophages.

Overall, our results suggest that the generation of M(LPS/IL4/ IL13) requires TLR4 and MyD88 signaling, resulting in downstream activation of $\mathrm{PI} 3 \mathrm{~K} / \mathrm{mTOR}$ and MAP kinases, and converging on phospho-CREB, STAT6, and NFE2. In addition, these cells have distinct transcriptional program and cell behavior.

\section{Signature of M(LPS/IL4/IL13) macrophages suggests a} phenotype conducive for repair responses

We mined the microarray data further and found prominent upregulation of transcripts in M(LPS/IL4/IL13) compared with all other conditions for several growth factors, including Hbegf (heparin binding epidermal growth factor), $P g f$ (placental growth factor), and the Flt1 gene that encodes vascular endothelial growth factor receptor-1 (Fig. 5a). Proteases that help in remodeling the lesion microenvironment for subsequent repair responses were also elevated in macrophages activated by LPS/IL4/IL13 compared with M (IL4/IL13) (Fig. 5b). In support, gelatin zymography of protein content for MMP-9 (Fig. 5c) and PCR analyses for Adamts4 (Fig. $5 d$ ), proteases important for matrix remodeling to support repair responses of the CNS (Larsen and Yong, 2004; Lemarchant et al., 2013), were elevated in M (LPS/IL4/IL13) compared with all other groups.

Further examination of the microarray data of M(LPS/IL4/ IL13) compared with M(IL4/IL13) macrophages found prominent elevation of regulatory cytokines, including IL1 receptor antagonist (Illrn), Il10, Il33, Il34, Il18, and for the Il4 and Il13 receptor subunits (Fig. 5e). The transcripts for the antioxidant enzyme superoxide dismutase and other molecules implicated in repair processes were also elevated in M(LPS/IL4/IL13) relative to M(IL4/IL13) macrophages (Fig. 5f).

We extended the analyses of HBEGF, an important growth factor for oligodendrocytes (Scafidi et al., 2014), by measuring its level in the conditioned media of macrophages through ELISA. 
Figure $5 g$ shows that HBEGF was markedly elevated in the secretome of M(LPS/IL4/IL13) cells, and that this prominent elevation was selective to this group.

Next, we investigated the metabolomic signature of M(LPS/ IL4/IL13) macrophages by subjecting their conditioned media to NMR spectroscopy as well as GC-MS. While lactate was readily produced in M(IFN/LPS) but not M(IL4/IL13) cells, the M(LPS/ IL4/IL13) generated a large quantity of this energy-related metabolite (Fig. 5h,i; Extended Data Fig. 5-1a,b). Remarkably, ornithine was massively elevated in the conditioned media of $\mathrm{M}$ (LPS/IL4/IL13) cells but not in all other groups (Fig. 5j; Extended Data Fig. 5-1c,d). Ornithine is considered an important repair amino acid as it feeds into the pathways of polyamine and proline synthesis that are important for tissue repair (Rath et al., 2014). Overall, the PLS-DA highlights the unique phenotype of M(LPS/IL4/IL13) cells (Extended Data Fig. 5-1e).

Specific metabolic modules regulate the activation of macrophages (Jha et al., 2015), and oxidative phosphorylation is more prominent in M2-like cells while M1-like cells are reported to be more glycolytic (Van den Bossche et al., 2015). We sought to investigate the metabolic state of M(LPS/IL4/IL13) by the Seahorse assay (Van den Bossche et al., 2015). We measured OCR (Fig. 5k) and ATP-dependent OCR (Fig. 5l), which reflect the mitochondrial function of the cells. OCR and ATP-dependent OCR were higher in M(LPS/IL4/IL13) and M(IL4/IL13) while lower in M(IFN/LPS) relative to control. M(LPS/IL4/IL13) used both glycolytic as well as oxidative phosphorylation pathways (Fig. $5 k, l$ ).

Lactate is an important trophic factor for OPC growth and differentiation (Ichihara et al., 2017) as is HBEGF, as noted earlier. Thus, to assess the consequence of elevation of HBEGF and lactate in M(LPS/IL4/IL13), we cultured murine OPCs (Keough et al., 2016) in medium devoid of growth factor supplements. We evaluated process outgrowth since this morphologic characteristic is important for the formation of multiple myelin internodes by oligodendrocytes in vivo. While control cells fared poorly in the growth factor-deprived condition, HBEGF alone increased the mean outgrowth per cell, and the combination of lactate and HBEGF further elevated process outgrowth (Fig. $5 \mathrm{~m}$, $n)$. In addition, HBEGF or HBEGF and lactate promoted the maturation of OPCs into MBP-positive oligodendrocytes (Fig. $5 m, o)$.

To further evaluate the metabolic pathways involved in activities of M(LPS/IL4/IL13), we used cell lysates from macrophages and processed the samples for mass spectroscopy (Fig. 6a) (Pride accession code: PXD15021). We corroborated earlier findings by proteomics analysis where proteomes of M(LPS/IL4/IL13) were elevated for TCA cycle (red), metabolic pathway (blue), and amino acid biosynthesis pathway (green) than those of M(IL4/ IL13) (Fig. 6b). Moreover, the mass spectroscopy analyses show that the M(LPS/IL4/IL13) cells elevated antioxidants, including glutathione metabolism (Fig. $6 c$ ), and had increased phagolysosome activity and thereby phagocytosis potential (Fig. $6 d$ ).

In summary, transcriptomic, metabolomic, and proteomic datasets reveal that LPS/IL4/IL13 triggers distinct properties in macrophages and suggest that they would contribute to repair in a lesion microenvironment.

\section{The application of the LPS/IL4/IL13 mixture to a demyelinating lesion modulates the activity of macrophages/ microglia}

We produced demyelination in the dorsal funiculus of the mouse spinal cord by the local deposition of the lipid disrupting toxin, lysolecithin (Fig. 7a). Three days later, a time point when macrophages and microglia were both abundant in the lesion as determined through a similar injury in $\mathrm{CX} 3 \mathrm{CR} 1^{\mathrm{GFP}}$ :CCR2 ${ }^{\mathrm{RFP}}$ mice (Yamasaki et al., 2014) (Fig. 7b), we reopened the suture and deposited $1 \mu \mathrm{l}$ of test solution locally (Fig. $7 c$ ). These solutions were saline for vehicle control, IFN/LPS, IL4/IL13, or LPS/IL4/IL13. We first characterized lesion size on day 7 of injury (i.e., $4 \mathrm{~d}$ after deposition of test solution), a time point associated with replenishment of OPCs and oligodendrocytes (Doring et al., 2015) (Fig. $7 d$ ). We found comparable demyelinated lesion size across all groups at the lesion epicenter (Fig. 7e); this was not unexpected since lysolecithin produces cell death and demyelination that is markedly extensive by $3 \mathrm{~d}$ of administration (Plemel et al., 2018). Finally, we found no treatment difference in accumulation of mononuclear phagocytes present at lesion sites, as revealed by pan macrophage/microglia marker Iba-1 and F4/80 staining (Fig. $7 f, g$ ).

We stained for phospho-CREB and phospho-STAT3/STAT6 in LPS/IL4/IL13-treated spinal cord to address the activation profile of macrophage/microglia but were unable to obtain reliable results; this approach was therefore aborted.

One recent finding suggests that pro-inflammatory $\mathrm{iNOS}^{+}$ macrophages change into the $\mathrm{Arg}^{+}$regulatory phenotype via an NNOS $^{+} \mathrm{Arg}^{+}$intermediate state in the spinal cord of EAE inflicted mice (Locatelli et al., 2018). It was also noted that iNOS $^{+} \mathrm{Arg}^{+}$macrophages could then convert to $\mathrm{Arg}^{+}$but not iNOS $^{+}$cells. In our study, the lesion of demyelinated mice administered vehicle did not have readily detectable proinflammatory $\left(\mathrm{iNOS}^{+}\right)$or regulatory $\left(\mathrm{CD} 204^{+}\right)$polarized macrophages/microglia (Fig. $7 g$ ) at day 7 of injury ( $4 \mathrm{~d}$ after vehicle deposition into the lesion). However, the lesion of IFN/LPSadministered mice had a prominent representation of iNOS $^{+}$ cells $($ Fig. $7 g, h)$. In demyelinated mice treated with IL4/IL13, despite the application of this strong regulatory/anti-inflammatory and putative reparative stimulus, CD204 expression was not readily apparent (Fig. $7 g, i$ ). Strikingly, the LPS/IL4/IL13 group manifested increased CD204, and mice did not have detectable NOS $^{+}$cells despite the presence of LPS in the test mixture (Fig. $7 g-i$ ). Thus, although the polarization of macrophages/microglia into a regulatory phenotype is not prominent after lysolecithin injury even with IL4/IL13, the presence of LPS/IL4/IL13 provided for a lesion microenvironment that was markedly CD $204^{+}$without incurring iNOS ${ }^{+}$pro-inflammatory neurotoxic cells. Using CX $3 \mathrm{CR} 1^{\mathrm{CreER} /+}$ :Rosa2 $6^{\text {tdTom/ } /+}$ reporter mice for microglia, we also observed the significant increase of Arg-1 expression in LPS/IL4/IL13 compared with saline group on both $\operatorname{tdT}^{+}$microglia (Fig. $7 j, k$ ) and CD45+ leukocytes (Fig. 7j,l; Extended Data Fig. 7-1).

\section{The application of the LPS/IL4/IL13 mixture to a}

\section{demyelinating lesion in mice accelerates oligodendrogenesis} We examined oligodendroglial lineage cells within the lesion by staining for the olig2 transcription factor common to these cells. Figure $8 a-c$ shows that there was an increase in olig $2^{+}$cells in the lesions of LPS/IL4/IL13-administered mice, and that this was associated with the elevated number of $\mathrm{CC}^{+}$mature oligodendrocytes as noted in a different experiment (Fig. 8b,d). The IL4/ IL13 administration was not adequate to elevate olig $2^{+}$or PDGFR $\alpha^{+}$Olig2 ${ }^{+}$OPCs, or $\mathrm{CC}^{+}{ }^{+}$Olig2 ${ }^{+}$mature oligodendrocytes. IFN/LPS treatment reduced the number of olig $2^{+}$and PDGFR $\alpha^{+}$Olig $2^{+}$OPCs suggestive of toxicity. Remarkably, the LPS/IL4/IL13 single local administration resulted $4 \mathrm{~d}$ after in elevated numbers of olig2 ${ }^{+}$lineage cells, and mature $\mathrm{CC}^{+}$Olig2 ${ }^{+}$ 
a

\begin{tabular}{|c|c|c|c|}
\hline Transcripts & M (IFN/LPS) & M (IL4/L13) & M (LPS/L4/LL13) \\
\hline Arg1 & 1.26 & 10.9 & 45.38 \\
\hline Flt1 & 3.07 & 11.59 & 29.60 \\
\hline Hbegf & 4.1 & 2.16 & 38.05 \\
\hline Pgf & 1.35 & 2.02 & 6.48 \\
\hline
\end{tabular}

e

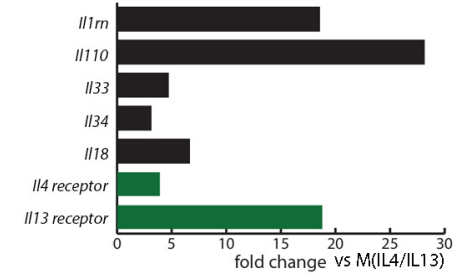

b

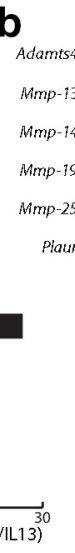

c

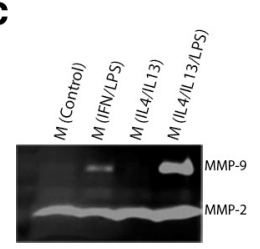

d

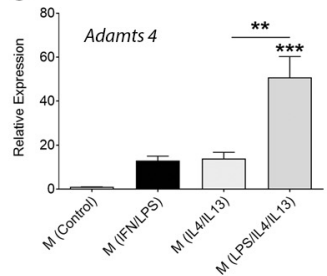

g

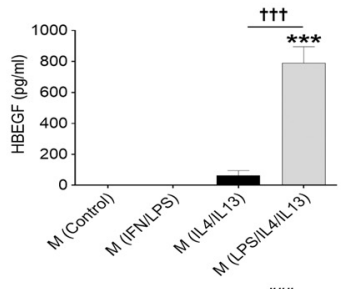

i

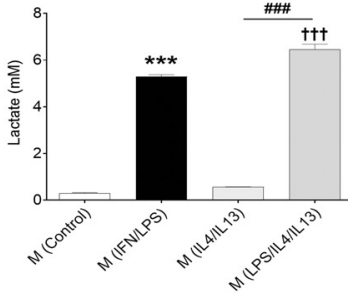

j

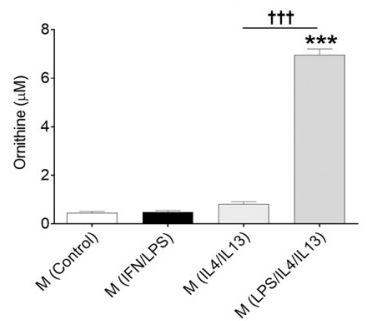

m
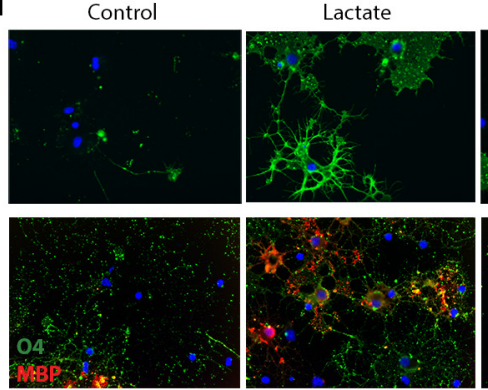

h

k

h $"$ RE

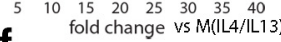

Arg- 1
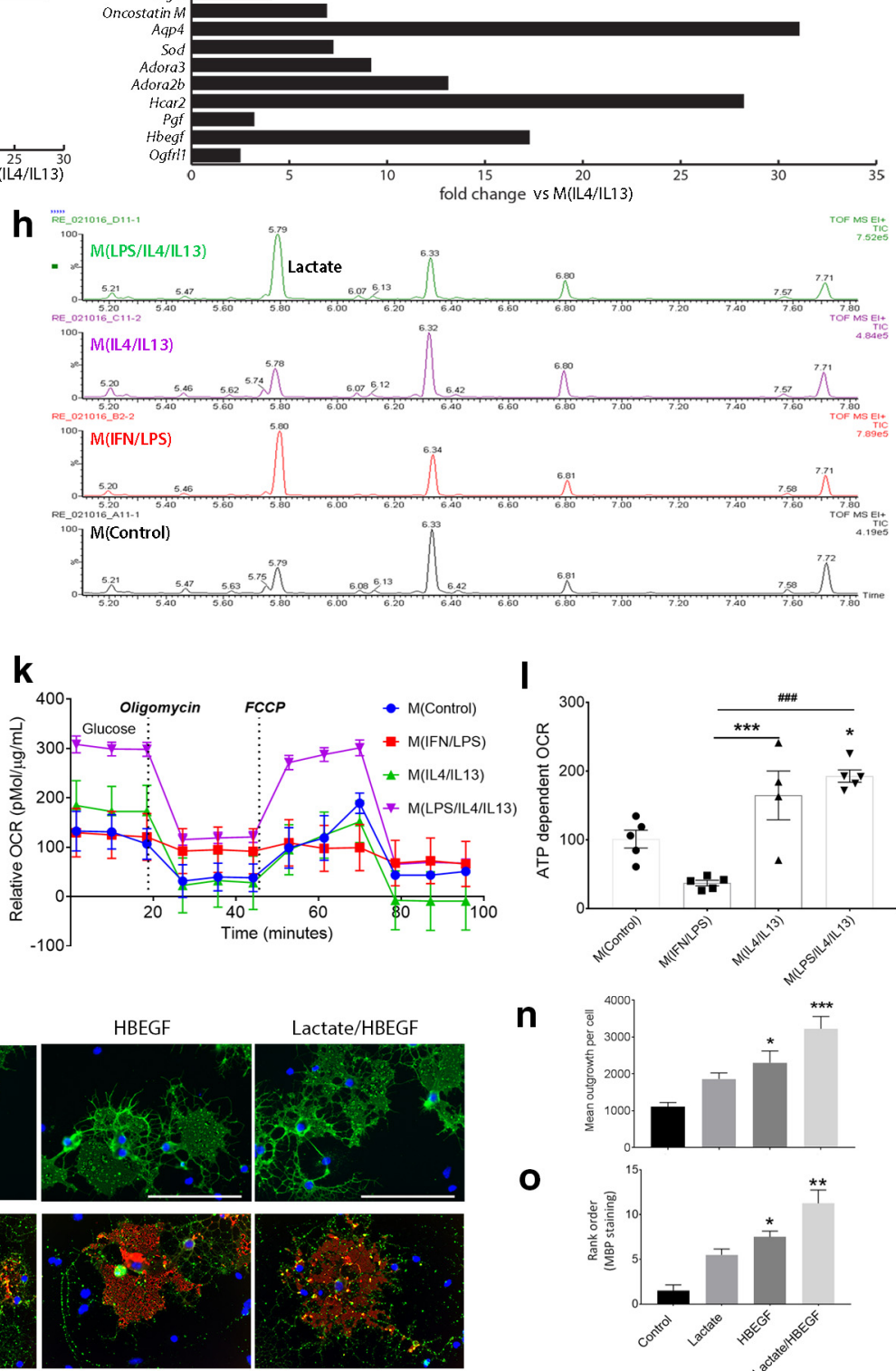

Lactate/HBEGF

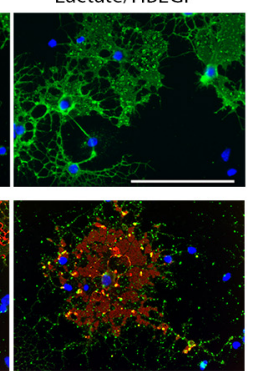

n

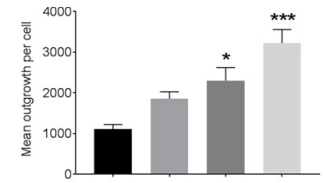

o

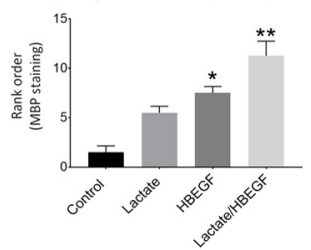

Figure 5. The omics signature of M(LPS/LL/IL13) suggests a phenotype conducive for repair responses. $\boldsymbol{a}$, Selected transcripts from microarray that were highly elevated in M(LPS/IL4/L13). $\boldsymbol{b}$, The fold change of transcripts of proteases elevated in M(LPS/L4/IL13) versus M(IL4/LL13). c, The high amount of MMP-9 in M(LPS/LL/IL13) was corroborated by gelatin zymogram and (d) by quantitative PCR for Adamts4 $\left(n=3 ; F_{(3,8)}=18.2 ;{ }^{* *} p=0.0038 ;{ }^{* * *} p=0.0005\right.$; one-way ANOVA, post hoc Tukey). $\boldsymbol{e}$, Transcripts for several regulatory cytokines and receptors for IL4 and IL13 were upregulated in M(LPS/LL/LL13), as are many enzymes and growth factors (f). As the microarray analyses that resulted in $\boldsymbol{a}, \boldsymbol{e}, \boldsymbol{f}$ were from two samples per group and where the results represent the mean of two samples, statistical comparisons have not been conducted as the $n$ of 2 per group would lack statistical validity. $\boldsymbol{g}$, M(LPS/L4/LL13) produced the highest amount of HBEGF $\left(n=3 ; F_{(3,8)}=48.86 ;{ }^{* * *} p=0.0001 ;+t+p=0.0001\right.$; one-way ANOVA, post hoc Tukey). Conditioned media from BMDMs were evaluated by GC-MS (h) and NMR spectroscopy (i,j). Cells activated with LPS, regardless of the presence or absence of IL4/LL13, had high amounts of $(\boldsymbol{h}, \boldsymbol{i})$ lactate $\left(n=3 ; F_{(3,8)}=741.8 ; * * * p=0.0001 ; \dagger+t p=0.0001 ;{ }^{\# \# \#} p=0.0001 ;\right.$ one-way ANOVA, post hoc Tukey), whereas (j) ornithine is elevated only in M(LPS/LL/IL13) $\left(n=3 ; F_{(3,134)}=571.8\right.$; ${ }^{* * *} p=0.0001 ;+t+p=0.0001$; one-way ANOVA, post hoc Tukey). The ornithine elevation in M(LPS/L4/L13) is emphasized in Extended Data Figure 5-1. M(LPS/L4//L13) and M(IL4/LL13) showed high OCR reflecting mitochondrial activity $(\boldsymbol{k}, \boldsymbol{I})$. $\boldsymbol{m}, 04$ staining for 0 PCs in growthdeficient medium at two different magnifications. Scale bar, $100 \mu \mathrm{m}$. $\boldsymbol{n}$, The combination of HBEGF and lactate for $24 \mathrm{~h}$ was very permissive for mean outgrowth of OPCs $\left(n=4 ; F_{(2,9)}=13.84\right.$; ${ }^{*} p=0.0368 ;{ }^{* * *} p=0.0014$; one-way ANOVA, post hoc Tukey) and also contributed to differentiation of OPC into MBP ${ }^{+}$oligodendrocytes $(\boldsymbol{m}, \boldsymbol{o})\left(n=4 ;{ }^{*} p=0.046 ;{ }^{* *} p=0.0024 ; \mathrm{Kruskal}-\right.$ Wallis test). 
a

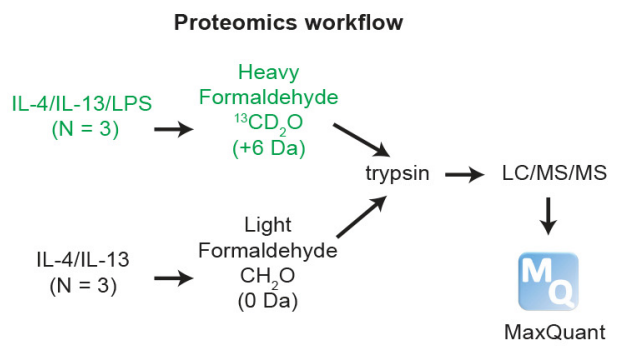

C

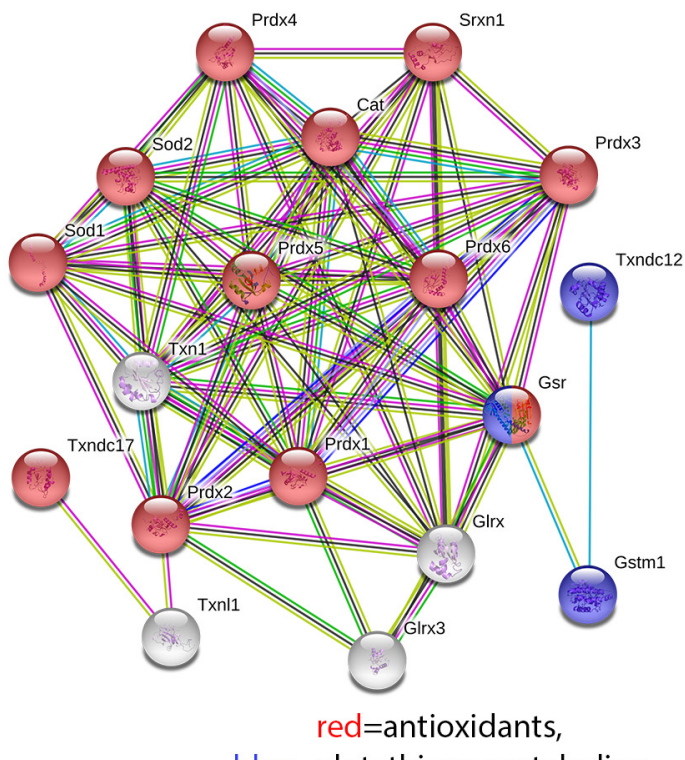

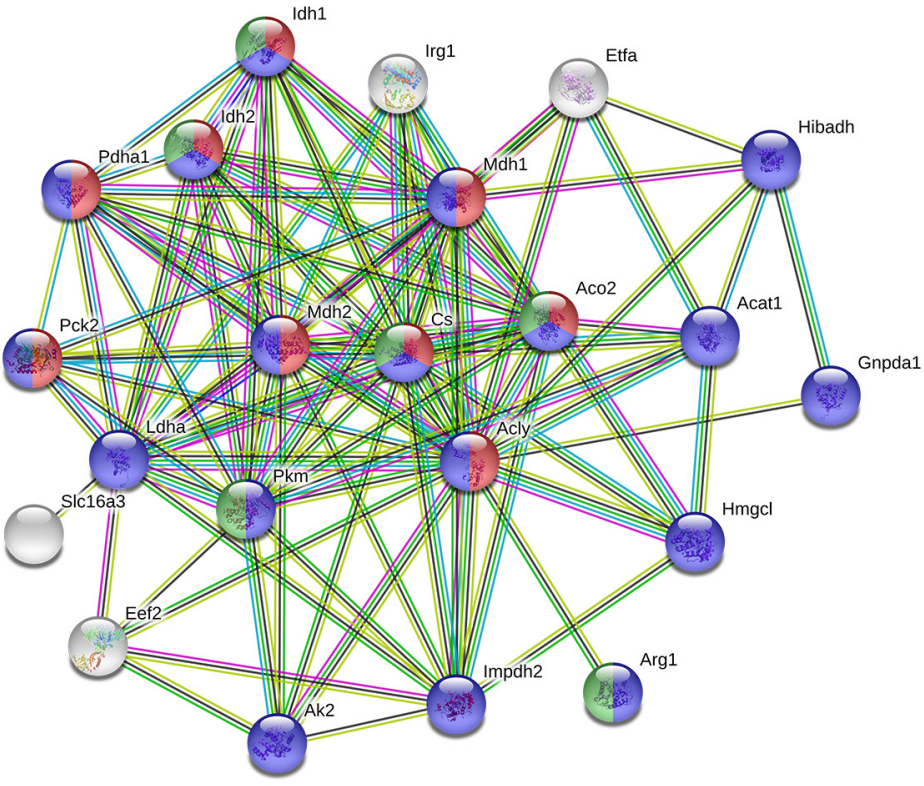

red=TCA cycle, blue=metabolic pathway, blue=glutathione metabolism

d Phagocytic machineries

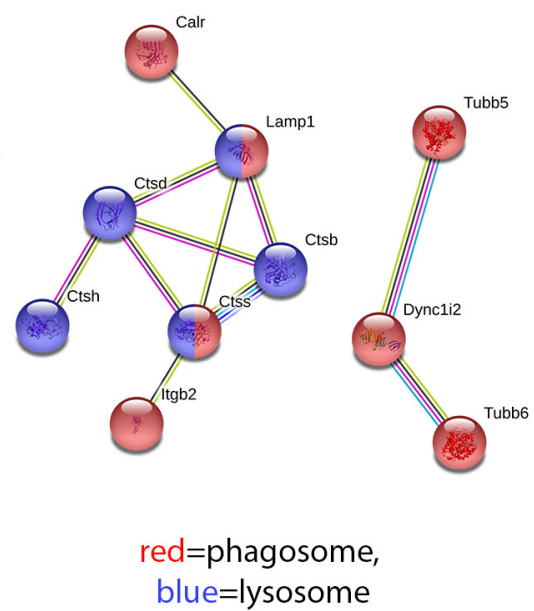

Figure 6. a, Proteomics workflow. Macrophages were activated for $24 \mathrm{~h}$; then cells were lysed and processed for proteomics ( $n=4$, for each condition). Data were obtained from LC/MS/ MS and were analyzed on MaxQuant. $\boldsymbol{b}$, Network analysis by STRING (https://string-db.org/) demonstrated the enrichment of molecules involve in tricarboxylic acid (TCA) cycle (red), metabolic pathway (blue), and amino acid biosynthesis (green) in M(LPS/IL4/IL13) group compared with M(IL4/IL13). c, Network analysis revealed the enrichment in antioxidant molecules (red) and proteins involve in glutathione metabolism (blue). $\boldsymbol{d}$, Further analysis determined increase in proteins involved in phagocytic machinery, such as phagosome formation (red) and lysosome increase (blue). Western blot detected changes to selected proteins are displayed in Extended Data Figure 6-1.

oligodendrocytes. The number of PDGFR $\alpha^{+}$Olig2 ${ }^{+}$OPCs was not different in the LPS/IL4/IL13 group compared with control, likely because most of the OPCs had differentiated into $\mathrm{CC1}^{+}$ oligodendrocytes.

\section{Mechanisms by which the LPS/IL4/IL13 treatment promotes a lesion microenvironment favorable for repair}

In vivo after a demyelinating injury, there is a requirement for macrophages for subsequent remyelination as their removal by silica quartz dust (Triarhou and Herndon, 1985) and clodronate liposomes (Kotter et al., 2005) or the abolishment of their infiltration into lesions in CCR2 null mice (Ruckh et al., 2012) impairs remyelination. Macrophages are required for the clearance of myelin debris and production of metabolites and growth factors for myelin repair (Miron and Franklin, 2014; Goldstein et al., 2016; Keough et al., 2016; McMurran et al., 2016). The observation that the M(LPS/IL4/IL13) macrophages are activated, migratory, and phagocytic in culture (Figs. 1,7), and produce remodeling proteases and growth metabolites (ornithine, HBEGF) (Fig. 5) would bode well for their remodeling of the lesion microenvironment necessary for remyelination. We examined the activity of macrophages/microglia within the lesion 
a

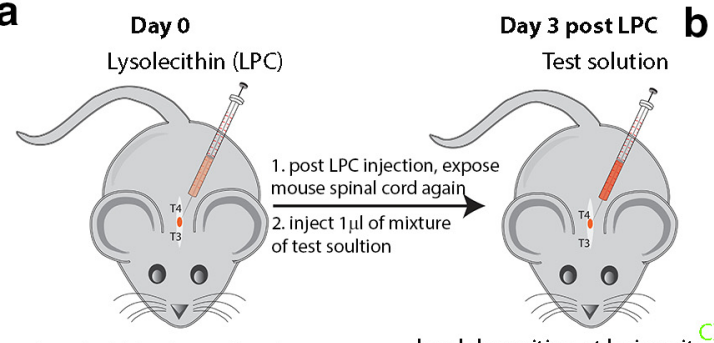

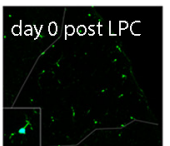

day 3 post LPC

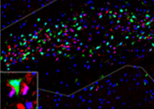

C Experimental timeline

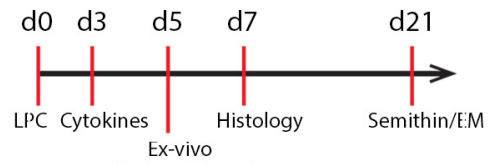

multiphoton imaging
Lysolecithin demyelination

local deposition at lesion site

3CR 1-GFP / CCR2-RFP

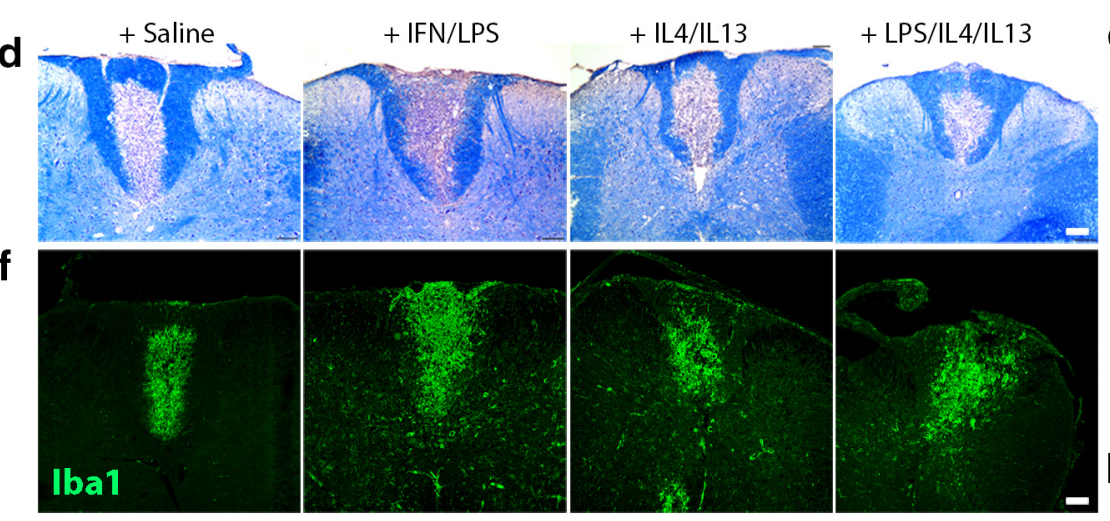

+ Saline

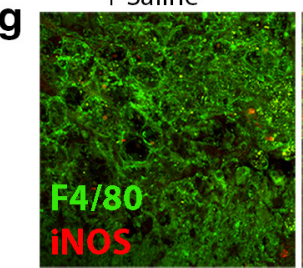

+ IFN/LPS
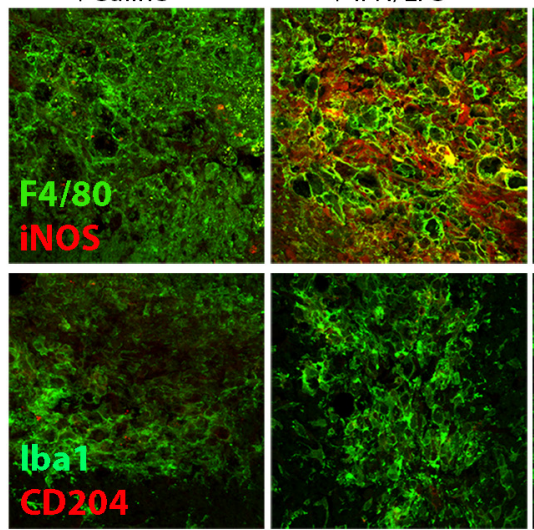

j

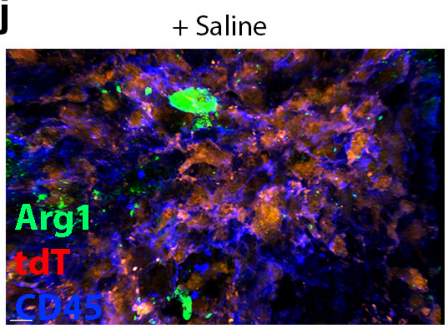

+ IL4/IL13
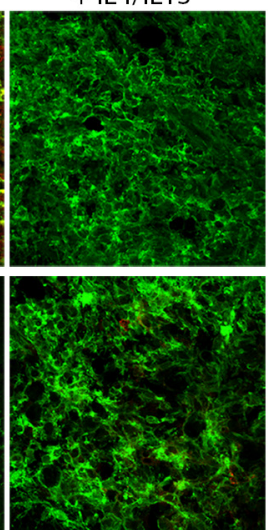

+ LPS/IL4/IL13

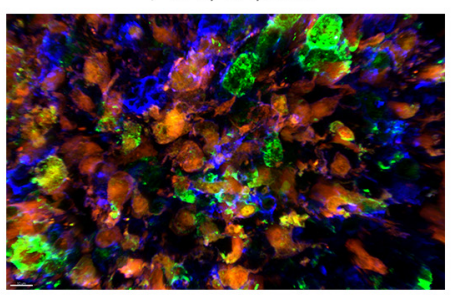

$+\llcorner P S / I L 4 / I L 13$
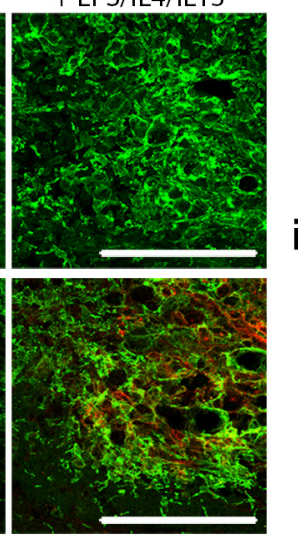

\section{i}

K

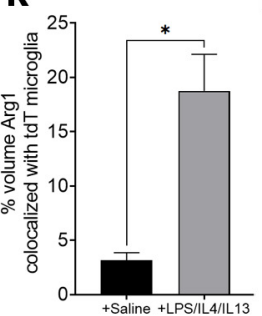

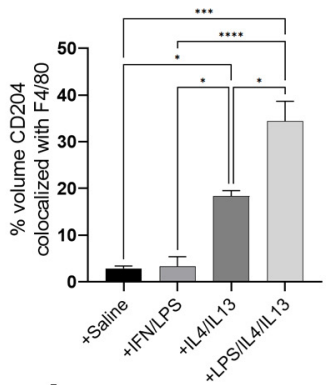

e
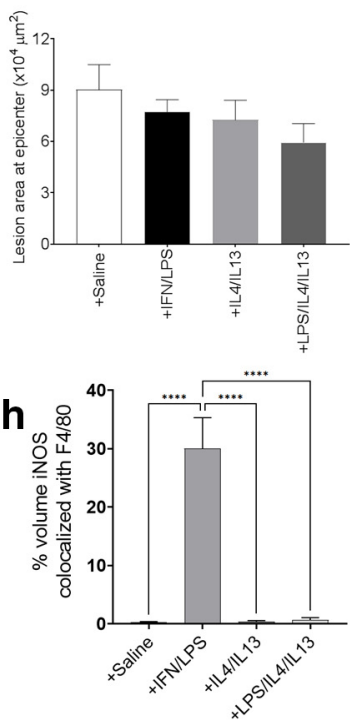

I

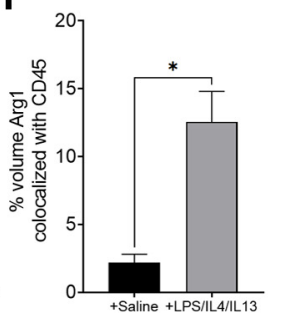

Figure 7. Experimental design and activation of macrophages/microglia in vivo. $\boldsymbol{a}$, The demyelination model whereby lysolecithin was locally deposited between T3 and T4 vertebrae in the dorsal column of the spinal cord of mice on day 0.0 day 3 , after reopening of the suture, $1 \mu$ of test solutions was locally deposited at the same place. $\boldsymbol{b}$, The day 3 time point was selected because the macrophage/microglia representation was increased at the lesion site as suggested by the presence of red/green cells (i.e., CCR2-RFP and CX3CR1-GFP, indicating mainly, but not exclusively, monocyte-derived macrophages and microglia, respectively). $c$, The experimental timeline for the in vivo experiment. Mice were killed $7 \mathrm{~d}$ following lysolecithin demyelination (and $4 \mathrm{~d}$ after local deposition of test solutions). $\boldsymbol{d}$, Representative images of 5 mice per group are displayed for eriochrome cyanine and neutral red-stained section where demyelination at $7 \mathrm{~d}$ is comparable across all the groups by one-way ANOVA (e). $\boldsymbol{f}$, Mice had similar extent of macrophages/microglia representation (Iba1) at the lesion site. $\boldsymbol{g}$, The staining within the lesioned dorsal column. IFN/LPS-treated mice had iNOS representation while more CD204 (but not iNOS) was found in LPS/LL/13-treated animals. Scale bar, $100 \mu \mathrm{m}$. $\boldsymbol{h}$, $\boldsymbol{i}$, Colocalization analyses were performed across the treatment group for iNOS and F4/80 $\left(n=3-5 ; F_{(3,12)}=34.08 ;{ }^{* * * *} p=0.0001\right.$; one-way ANOVA, post hoc Tukey) and also for CD204 and Iba1 ( $n=3-5 ; F_{(3,11)}=25.4 ;{ }^{*} p$

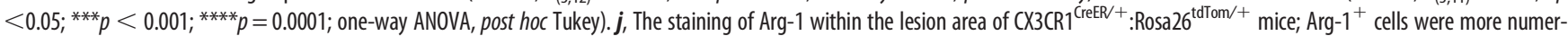
ous in LPS/L4/LL13-treated group and localized to ( $k$ ) microglia $\left(n=3\right.$ or 4 ; $\mathrm{df}=5 ;{ }^{*} p=0.0117$; two-tailed $t$ test) and (I) monocyte-derived macrophages $\left(n=3\right.$ or 4; $\mathrm{df}=5$; ${ }^{*} p=0.0121$; two-tailed $t$ test). Other examples of high-magnification images with IMARIS rendering to present iNOS and Arg-1 within macrophages and microglia in the lesioned spinal cord are displayed in Extended Data Figure 7-1. 
a

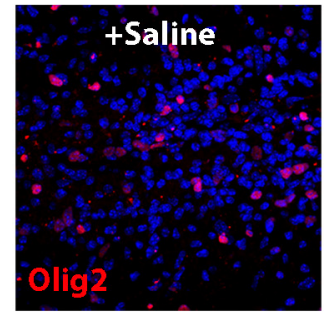

b

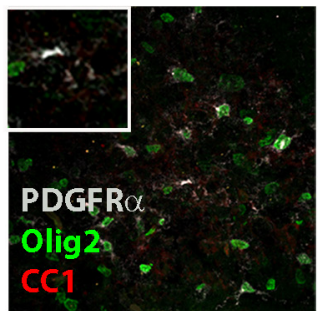

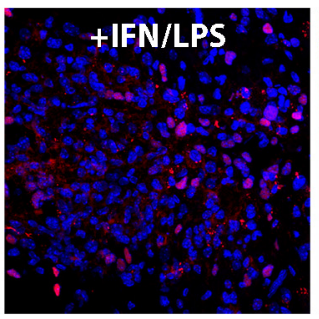
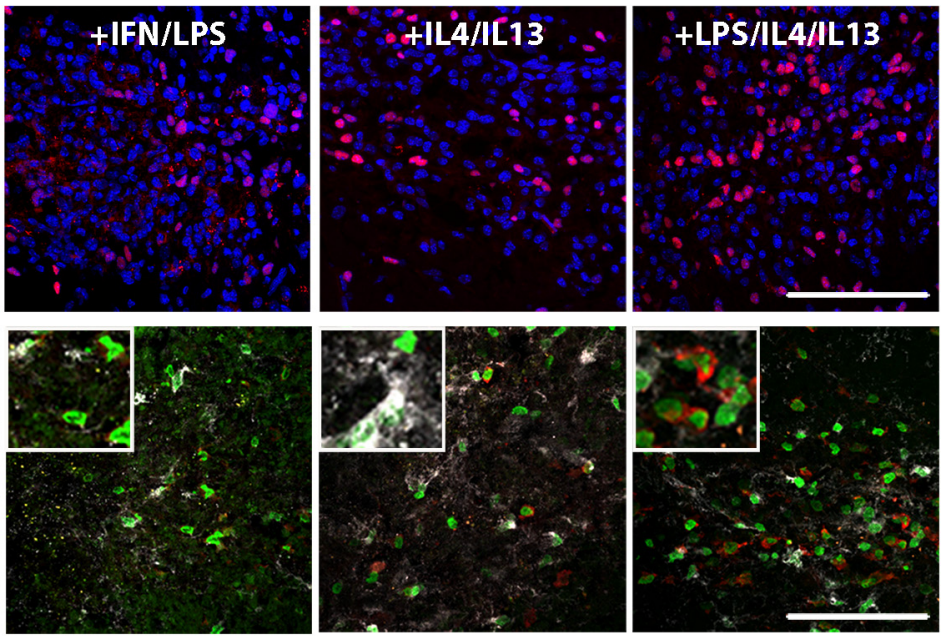

C

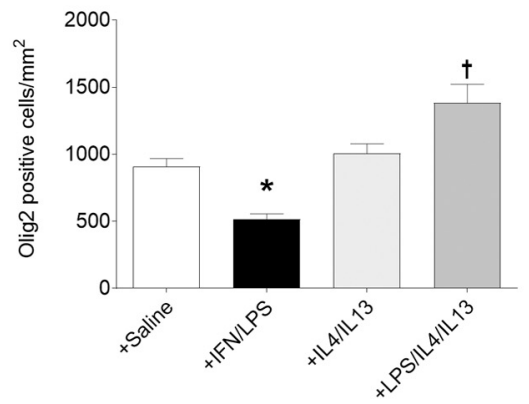

d

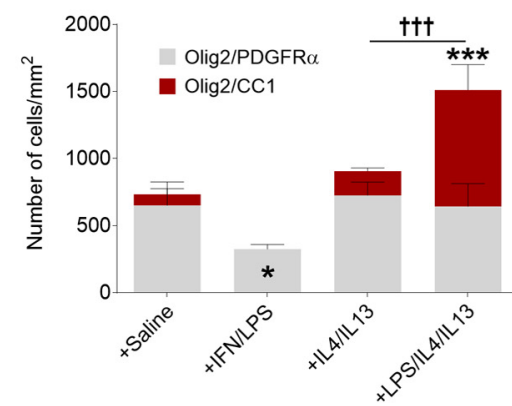

Figure 8. The application of the LPS/IL4/IL13 mixture to a demyelinating lesion in mice promotes oligodendrogenesis. $\boldsymbol{a}$, Cells of the oligodendrocyte lineage were quantified at lesion site 7 $\mathrm{d}$ after demyelination using immunohistochemistry for olig2 (red) and nuclear yellow (blue). $\boldsymbol{a}, \boldsymbol{c}$, Number of olig2-positive cells was quantified across the different treatment groups at the lesion site in the dorsal column $\left(n=3 ; F_{(3,8)}=17.14,{ }^{*} p=0.0487 ; t p=0.0190\right.$; one-way ANOVA, post hoc Tukey). Scale bar, $100 \mu$ m. $\boldsymbol{b}, \boldsymbol{d}$, The triple staining of olig2 (green), PDGFR $\alpha$ (marker of precursor cells, gray), and CC1 (mature oligodendrocytes, red) shows that the CC1 representation was highest in LPS/IL4/13 group ( $n=4$; ${ }^{*} p=0.0126 ;{ }^{* * *} p=0.0001$; $++\dagger p=0.0001$; one-way ANOVA, post hoc Tukey). Scale bar, $100 \mu \mathrm{m}$

through imaging of the spinal cord (Fig. $9 a-g$ ) using real-time ex vivo multiphoton microscopy (Stirling et al., 2014; Plemel et al., 2018; Rawji et al., 2018). Given the technical challenges, we focused on the saline and LPS/IL4/IL13 groups. We found CX3CR1-GFP cells to have low- and high-fluorescence intensity, the significance of which is unknown but may be related to the activation state of cell, so we evaluated our results accordingly. Figure $9 c$ depicts that both populations trended toward an increase in the LPS/IL4/IL13-treated group compared with vehicle; the surface area and volume as indicators of macrophage/ microglia activity were significantly more in the LPS/IL4/IL13 group (Fig. 9d,e). In addition, these activated cells had higher speed and mean track displacement that suggest their increased capacity (Plemel et al., 2018) to survey the lesion site (Fig. 9f,g).

The presence of myelin debris is a major impediment for remyelination (Kotter et al., 2005). To assess myelin phagocytosis in vivo, we quantified $\mathrm{GFP}^{+}$cells for their intracellular Nile Red content as a reflection of phagocytosis and clearance of myelin debris. The $\%$ of $\mathrm{GFP}^{+}$cells that were phagocytic (with Nile red, regardless of amount) trended toward an increase after LPS/IL4/ IL13 treatment (Fig. 9h,i). Within each phagocyte that has engulfed myelin debris, the Nile Red content was significantly higher in the LPS/IL4/IL13 group over that of vehicle-treated mice (Fig. 9h-j).

Another major impediment to remyelination is the accumulation of the versican chondroitin sulfate proteoglycans in lesions (Keough et al., 2016). When sections were stained for versican
$\mathrm{V} 1$ at day 7 after injury, the expression of V1 was least in the LPS/IL4/IL13-treated group (Fig. 9k,l). Along with the data that the M(LPS/IL4/IL13) macrophages produce high amounts of ECM-degrading proteases (Fig. 5), these results suggest that treatment with LPS/IL4/IL13 generated a macrophage state within demyelinated lesions that efficiently remove accumulated chondroitin sulfate proteoglycans.

Metabolic demand increases during remyelination, and lactate is a major energy source to promote differentiation of OPCs (Ichihara et al., 2017). To assess metabolites within lesions, we subjected spinal cord ventral hemisections to GC-MS and found significant elevation of several metabolites, including lactate in the LPS/IL4/IL13 group compared with that of saline treatment (Fig. $9 \mathrm{~m}$ ). The OPLS-DA (orthogonal projections to latent structures discriminant analysis) evaluation highlighted that the vehicle and LPS/IL4/IL13-treated mice were clearly two distinct groups based on metabolites (Fig. 9n). Finally, we assessed the amount of HB-EGF within demyelinated tissue lysates by ELISA and found that, while injury did not elicit a rise of detectable HB-EGF at day 7 compared with that in naive animals, the deposition of LPS/IL4/IL13 produced a marked increase of this growth factor within lesions (Fig. 9o).

\section{The LPS/IL4/IL13 treatment promotes remyelination}

We determined whether the crucial outcome of remyelination is promoted by the cytokine mixture administered once at day 3 
a

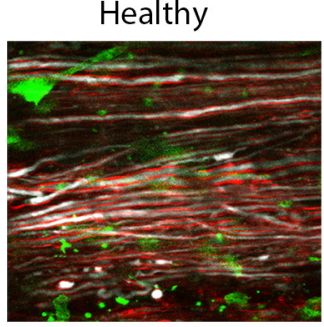

C

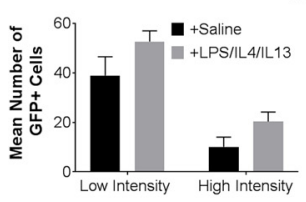

+Saline

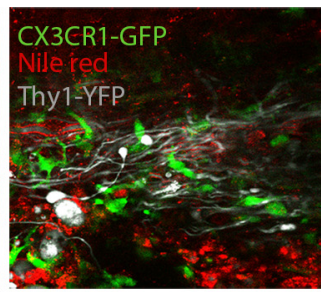

e

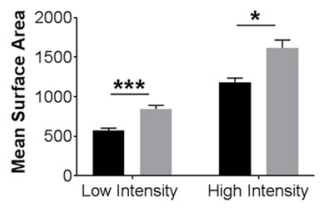

+LPS/IL4/IL13
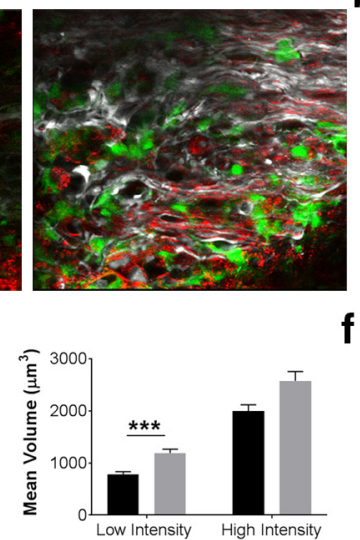

f b
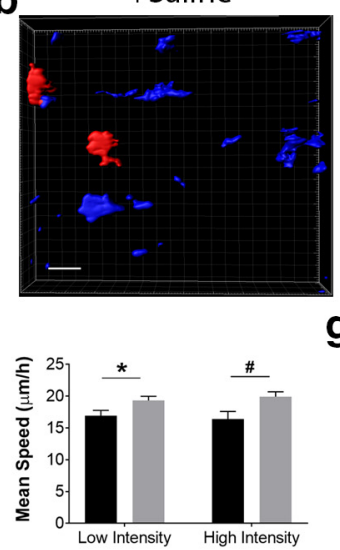

+LPS/IL4/IL13

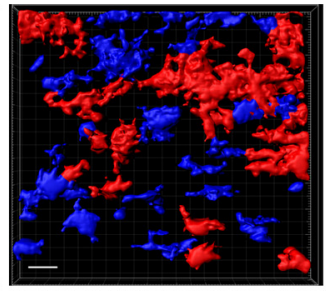

g

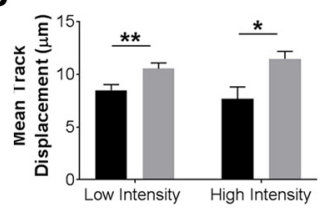

h
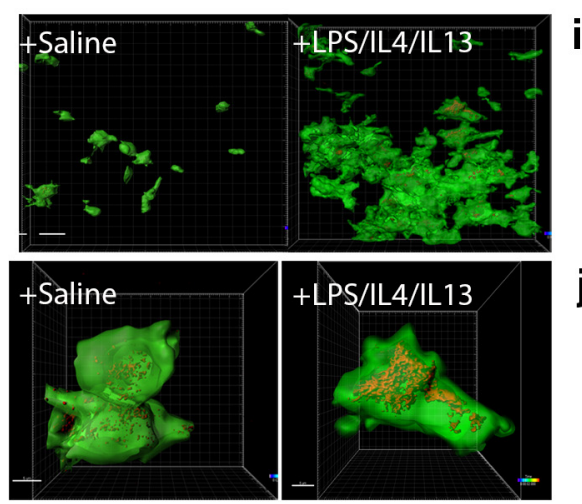
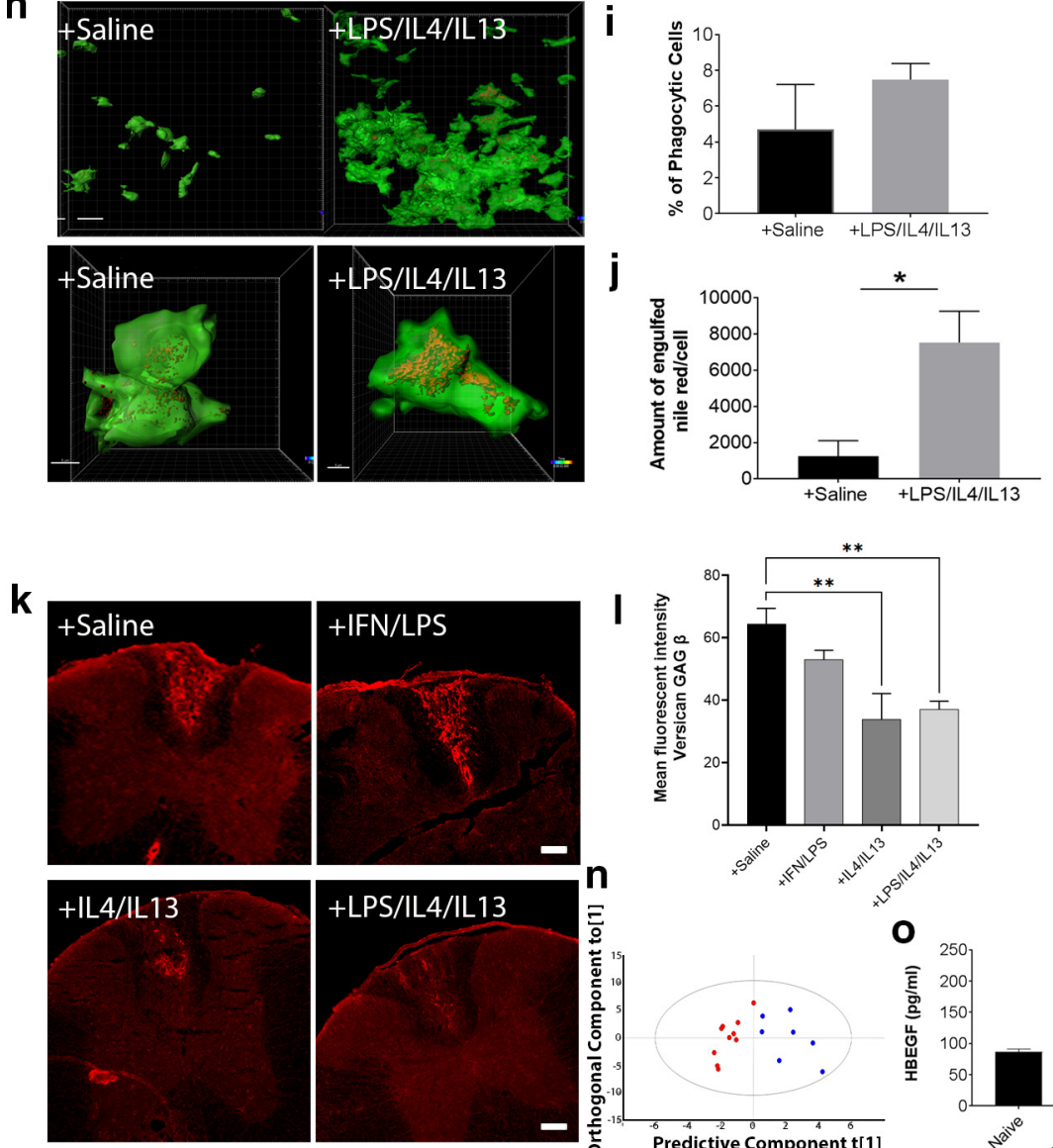

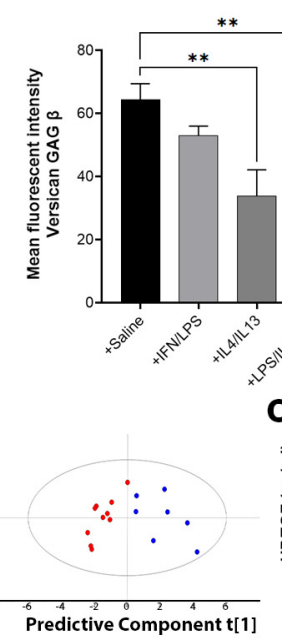

m Coefficient CS [1] (LPS/IL4/IL13)
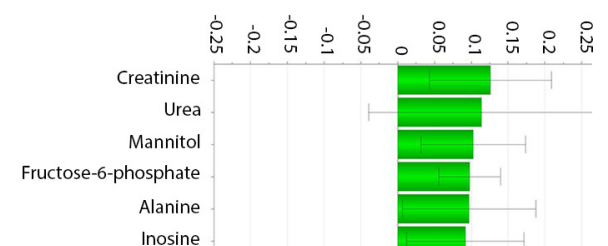

Galactose-6-phosphate

Glutamic acid

Glucose 6-phosphate

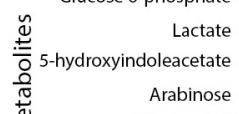

Arabinose
Syllo-Inositol

GABA

Threonine

Glycyl-glutamic acid

Glucose

Serine

Sugar Deriv 6

Arginine

Galactose

Mannose

Phenylalanine

Myristic acid

Adenine

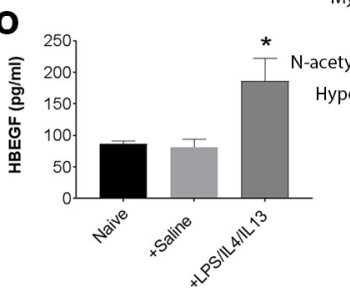

tyl-arginine

Purine

Figure 9. Characteristics of the lesion microenvironment following LPS/L4/IL13 treatment. $\boldsymbol{a}$, Ex vivo real-time imaging of the spinal cord of $C \times 3 c r 7^{6 F P /+}:{\text { Thy } 7 Y F P P^{+}}^{+}$mice with myelin labeled by Nile Red. $\boldsymbol{b}, \mathrm{CX}_{3} \mathrm{CR} \mathrm{T}^{\mathrm{GP} /+}$ macrophages in lesions have high- or low-intensity signal, and these were quantitated within lesions. Scale bar, $20 \mu \mathrm{m}$. Only demyelinated mice administered saline or LPS/L4/L13 within lesions were examined because of the complexities of these experiments. There was a trend toward an increase in the mean number of macrophages/microglia (c) following LPS/L4/IL13 treatment, and the latter elicited GFP ${ }^{+}$cells that were larger $(\boldsymbol{d}, \boldsymbol{e})$ and were more migratory $(\boldsymbol{f}, \boldsymbol{g})$, depicting surveillance activity (Plemel et al., 2018), within the area of demyelination $\left(n=38-100, t\right.$ test stats). ${ }^{*} p<0.05 ;{ }^{* *} p<0.01 ;{ }^{* * *} p<0.001 ;{ }^{*} p<0.05$. Imaris surface rendering and analysis of Nile Red within cells as an index of phagocytosis (scale bars: $20 \mu \mathrm{m}$; zoomed in image, $5 \mu \mathrm{m}$ for) (h) shows that the percentage of macrophages/microglia that have engulfed myelin debris is similar (i) between saline and LPS/IL4/IL13 treatment, but that the latter promoted the extent of myelin engulfment/phagocytosis $(j)$ within macrophages/microglia $\left(n=3 ; t=3.242, \mathrm{df}=4, F_{(2,2)}=4.117 ;{ }^{*} p=0.0316\right.$; unpaired $t$ test, two-tailed). $\boldsymbol{k}$, Staining for versican V1 $2 \mathrm{~d}$ after treatment (d 5 after demyelination) shows deposition of this inhibitor of OPCs (Keough et al., 2016) within lesions in all groups, but expression was least in the LPS/L4/IL13 group, indicating either reduced deposition or enhanced removal. Scale bar, $100 \mu \mathrm{m}$. $I$, The mean fluorescence intensity was quantified across the treatment group for versican V1 $\left(n=3-5 ; F_{(3,11)}=9.36 ;{ }^{* *} p<0.01\right.$; one-way ANOVA, post hoc Tukey). The dorsal half of a $3 \mathrm{~mm}$ coronal block of spinal cord containing the demyelinated dorsal column from saline or LPS/IL4/IL13 groups was homogenized for GC-MS analysis (m); OPLS-DA score scatter plot shows the two groups to be different (red represents LPS/L4//L13; blue represents saline; each circle represents a different mouse, $\mathrm{R} 2=0.801, \mathrm{Q} 2=0.667$ ) $(\boldsymbol{n})$. The differential metabolites displayed in I show elevation (right shift) or reduction (left). Finally, tissue homogenates subjected to HBEGF ELISA showed that LPS/IL4/L13 increased this growth factor $(\boldsymbol{o})$ within lesions $\left(n=5 ; F_{(2,11)}=4.73 ;{ }^{*} p=0.0265\right.$; one-way ANOVA, post hoc Tukey). All histograms are mean \pm SEM. 


\section{a Semithin sections}
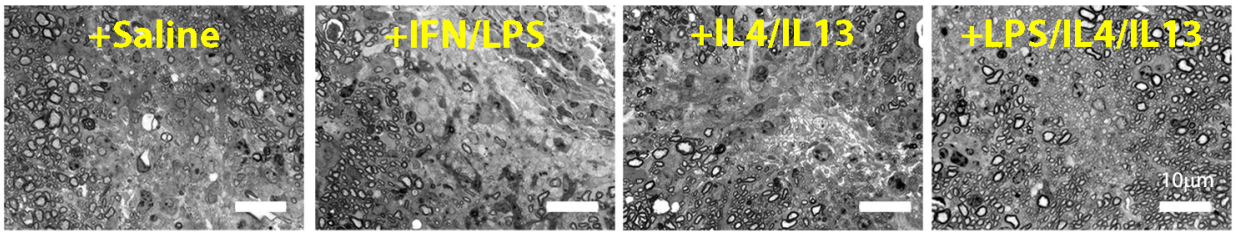

b Electron micrographs
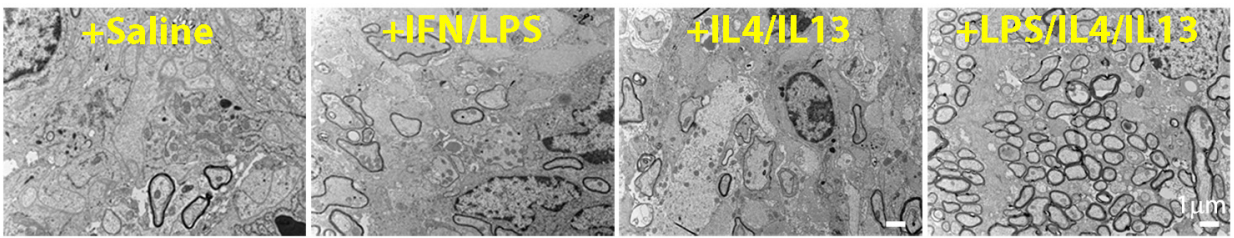

C

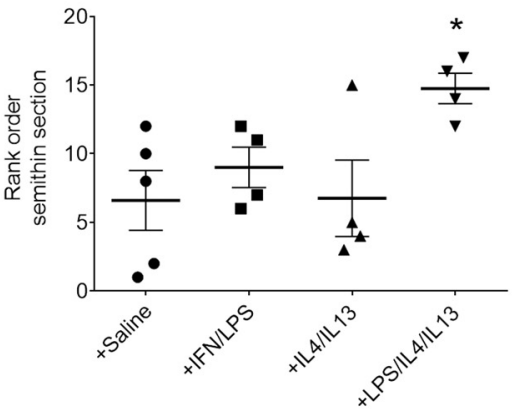

e

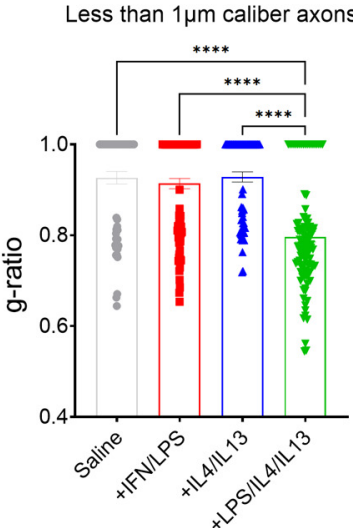

$1 \mu \mathrm{m}-2 \mu \mathrm{m}$ caliber axons

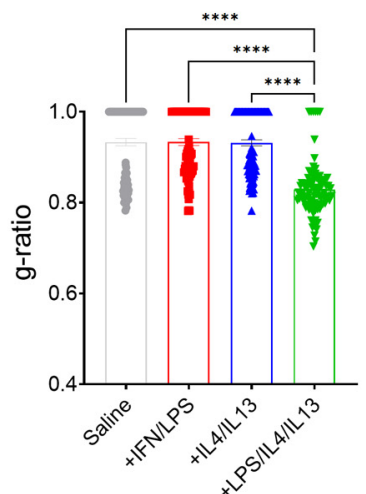

d

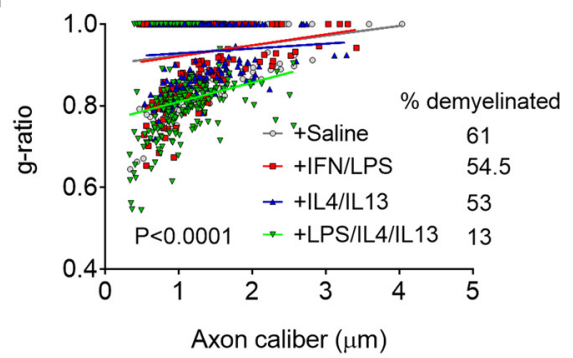

Figure 10. The LPS/LL/IL13 treatment promotes remyelination. $\boldsymbol{a}, A$ cross-sectional toluidine blue-stained semithin section of the dorsal column of white matter (scale bar, $10 \mu \mathrm{m})$ at $\mathrm{d} 21$ following lysolecithin injury shows more remyelinated profile in LPS/IL4/L13-treated group, and this was corroborated by electron micrographs. Scale bar, $1 \mu \mathrm{m}$. $\boldsymbol{b}$, c, Blinded rank order analysis ( $n=4$, one-way ANOVA, Kruskal-Wallis test; ${ }^{*} p=0.0481$ ) of toluidine blue-stained semithin sections document semiquantitatively the highest degree of remyelination in LPS/IL4/L13 treatment group. $\boldsymbol{d}, 200$ axons were counted per treatment group to confirm remyelination. The most abundant remyelination was found in electron micrographs of LPS/L4/LL13 treatment where the $\%$ axons remaining demyelinated was least, and this was verified by g-ratio analyses of remyelinated axons where in linear regression, the lower the $y$ intercept, the thicker the myelin sheath (linear regression, $\left.F_{(3,795)}=79.66, p<0.0001\right)$. $\boldsymbol{e}$. We further analyzed the g-ratio based on binning of the axon diameters in three categories $(<1,1-2$, and $>2 \mu \mathrm{m})$. The effect of LPS/L4/LL13 on g-ratio (myelin thickness) was significant across axons of all diameters. ${ }^{*} p<0.05$; ${ }^{* *} p=0.01$; ${ }^{* * *} p<0.00001$; Tukey's multiple comparisons test.

after injury (as depicted in Fig. 7c). Mice were killed at day 21 to allow time for oligodendrocytes to reform compact myelin around axons. Semithin epon sections stained for toluidine blue were subjected to blinded semiquantitative rank order evaluation of extent of remyelination. The animals with the highest amount of remyelination profiles tended to be those in the LPS/IL4/IL13 treatment group (Fig. 10). To confirm this, sections were taken for electron microscopy. Within the lesion area, the number of axons with thin myelin that is indicative of remyelination was visibly higher and widespread in the LPS/IL4/IL13 group; blinded rank order analysis of semithin bright field images corroborated that remyelination profiles were most abundant in the LPS/IL4/IL13 treatment group (Fig. 10a-c). This was verified by g-ratio analysis of individual axons (Fig. 10d), which also demonstrated that the LPS/IL4/IL13 resulted in axons that had thicker myelin (lower g-ratio) than axons in all the other groups; we did not evaluate quantitatively whether axons of all diameter had thicker myelin sheath as a result of mixture treatment. The g-ratio analysis also informs that, despite the IL4/IL13 mixture being a putative reparative mixture, its single administration is inadequate to promote remyelination beyond that of vehicle control. In contrast, the addition of LPS to the IL4/IL13 mixture led 
to profound remyelination as manifested by the g-ratio and by the $13 \%$ of axons remaining demyelinated in contrast to the 53\%-61\% still demyelinated in the other three groups (Fig. 10d). The effect of LPS/IL4/IL13 on remyelination was observed for axons of diameters $<1,1-2$, and $>2 \mu \mathrm{m}$ (Fig. 10e).

\section{Discussion}

Blood-derived macrophages originate as circulating monocytes that migrate into sites of injury where they fulfill diverse functions, including phagocytosis of debris, provision of various growth factors, and scar formation (Raposo and Schwartz, 2014; Ransohoff, 2016; Prinz and Priller, 2017). Activated macrophages/microglia within the CNS were considered detrimental to CNS well-being; but over the past two decades, research has revealed their multiple roles in protection against neurodegeneration and in CNS repair (Kigerl et al., 2009; Mei et al., 2016; Lloyd and Miron, 2019). A large body of literature now describes that the depletion of circulating monocytes impairs attempts at CNS remyelination and axonal regeneration (Kotter et al., 2005); conversely, promoting functions of macrophages/microglia within the injured CNS can enhance neuroprotection, axonal outgrowth, and remyelination (Miron et al., 2013; Doring et al., 2015; McMurran et al., 2016; Rawji et al., 2020). Thus, the challenge is how to mobilize macrophages/microglia for reparative outcomes without eliciting their potential harmful effects.

It is now well established that macrophages/microglia can exert a number of distinct activities depending on the priming and activation stimuli in its microenvironment (Xue et al., 2014). The classic TL4 agonist, LPS, particularly in combination with the priming stimulus IFN- $\gamma$, is often associated with activation of macrophages along a pro-inflammatory and potential harmful state, whereas IL4/IL13 is often used to drive macrophages along a regulatory, anti-inflammatory, and reparative type (Mantovani et al., 2004; Kigerl et al., 2009; Durafourt et al., 2012; Miron et al., 2013). Herein, we made the surprising finding that the addition of LPS to IL4/IL13 results in a previously undescribed and particularly reparative state of macrophages/microglia. The M(LPS/ IL4/IL13) or $\mathrm{Mi}(\mathrm{LPS} / \mathrm{IL} 4 / \mathrm{IL} 13)$ cells were without worrisome features of LPS, such as the generation of pro-inflammatory IL12 or neurotoxic roles; they exceeded the properties of IL4/IL13treated cells, such as chemotaxis and the production of HB-EGF, lactate, ornithine, and other metabolites. Following lysolecithin demyelination, the LPS/IL4/IL13-treated spinal cord microenvironment significantly elevated the number of oligodendrocytes and robustly promoted remyelination. In contrast, the lesion microenvironment, even with delivery of IL4/IL13, was insufficient to foster a reparative milieu beyond that occurring spontaneously after injury.

The mechanisms of the M(LPS/IL4/IL13) macrophages/ microglia for repair, including the thicker myelin sheath, likely encompass additional features that are not mutually exclusive: production of proteases such as MMP-9 and ADAMTS4 that can degrade negative regulators of remyelination such as versican, the efficient phagocytosis, and clearance of inhibitory myelin debris, metabolic and antioxidant activities, and the generation of the regulatory cytokine IL10 that has been found to temper a pro-inflammatory milieu to enable repair (Shechter et al., 2009). We note that the transcripts encoding receptors for IL4 and IL13 are also elevated in M(LPS/IL4/IL13), potentially creating a positive loop for a more efficient IL4 and IL13 signaling. The local amplification of IL4R $\alpha$-mediated macrophage activation has been reported to promote repair (Fenn et al., 2014; Francos-
Quijorna et al., 2016). Moreover, IL4 can activate the IL4 receptor on neurons and plays an important role in direct neuroprotection (Walsh et al., 2015). It has also been shown that alternatively activated macrophages secrete the IL4-associated enzyme, IL4i, that increases CNS remyelination and axonal protection (Psachoulia et al., 2016).

Our results highlight the unexpected integration of proinflammatory (LPS) and anti-inflammatory (IL4/IL13) triggers with predominance of the latter cue, since the phenotype of $\mathrm{M}$ (LPS/IL4/IL13) can still be generated with IL4/IL13 administered $12 \mathrm{~h}$ after the LPS. The generation of the reparative phenotype requires MyD88 but not TRIF signaling, and this is corroborated by the inability of monophosphoryl lipid A, a truncated LPS that affects TRIF but not MYD88 (Michaud et al., 2013) to elicit the macrophage state produced by LPS. Recently, MYD88 signaling was also implicated as favoring remyelination in zebrafish and mice (Cunha et al., 2020). We determined that LPS acting through TLR4 can be substituted to some extent by the TLR2 agonist, PAM. Downstream to these receptors and signaling adaptors, the LPS/IL4/IL13 exposure engaged both p-STAT6 and $\mathrm{p}$-CREB, which differentiated it from IFN/LPS that triggered $\mathrm{p}$ CREB but not p-STAT6, and from IL4/IL13 that activated pSTAT6 but not p-CREB.

Furthermore, results from ChIP-seq experiments provided evidence that costimulation modifies activity of signaling pathways and transcription factors modulated by LPS and IL4/IL13. These results are in agreement with a previously published study that demonstrated that complex immune stimulation of macrophages leads to significant cross-talk and synergistic activity of transcription factors at the genomic level (Eichenfield et al., 2016). Finally, de novo motif enrichment analysis performed on genomic regions that display higher abundance of $\mathrm{H} 3 \mathrm{~K} 27 \mathrm{ac}$ following LPS/IL4/IL13 costimulation compared with any other conditions suggested that NFE2 transcription factor, which is also known as NRF2, may be a key effector of these synergistic signaling activities. Interestingly, we note that NFE2 and CREB1, which our gene profiling analysis also implicated as a potential driver of the LPS/IL4/IL13 transcriptional program, have previously been shown to collaborate with one another in the transcriptional regulation of endothelial cells during inflammatory events (Mylroie et al., 2015). Collectively, these observations suggest that LPS/IL4/Il13 costimulation may promote a distinct profile of cooperative activity between NFE2 and CREB1 in macrophages. In turn, such collaborative transcriptional activity specifies a gene expression program that enables these cells to acquire very high tissue repair and regeneration capabilities.

The phenomenon of LPS preconditioning, whereby a prior exposure to LPS leads to reduced pathophysiology on a subsequent insult, has been described for some time (Turner et al., 2017). Whether the mechanism of benefit of LPS preconditioning relates to the generation of reparative and protective macrophage populations similar to that seen for M(LPS/IL4/IL13) is a possibility to be explored in future studies. In a recent work (Torres-Espin et al., 2018), the authors found eliciting inflammation by LPS, together with exercise help in rehabilitation for chronic spinal cord injury. The mechanism behind this effect is not known, but the outcome is encouraging and interesting.

The current study has identified a state of macrophages and microglia that is particularly reparative; and where the cytokine and LPS stimuli used could potentially be applied locally in focal injuries, such as traumatic brain and spinal cord injuries. However, the activators would not be conducive for systemic application, although LPS can be found in the circulation, 
including in subjects with the demyelinating disease multiple sclerosis (Teixeira et al., 2013). A limitation herein, and a challenge going forward, would be to identify therapeutics that stimulate the downstream pathways unleashed by the LPS/IL4/IL13, so as to produce these remarkable macrophages that can be helpful in a variety of neurologic and non-neurologic conditions.

Another limitation of this study was the inability to discriminate monocyte-derived macrophages from microglia; we thus resorted to using "macrophages/microglia" when describing these cells. The $\mathrm{CX} 3 \mathrm{CR} 1^{\mathrm{CreER} /+}:$ Rosa $26^{\mathrm{tTom} /+}$ mouse is a step forward for immunofluorescence differentiation of monocytederived macrophages from microglia, but this does not provide enough cells for the biochemical and molecular profiling studies within. Moving forward, novel tools, such as the HexB reporter mice (Masuda et al., 2020), could be used for microglia while a binary Cre transgenic system enables differentiation of microglia from brain-resident macrophages, such as perivascular macrophages (Kim et al., 2021).

In conclusion, through the unconventional application of LPS along with IL4/IL13, we have uncovered a previously unappreciated, and particularly reparative, state of macrophages/microglia. Our emphasis is not whether this state of macrophage/microglia exists in vivo normally or during recovery from neural injury, but rather that they can be pharmacologically generated by the cytokine mixture of LPS/IL4/IL13. Learning how to mobilize these reparative macrophages/microglia should pave the way toward beneficial inflammation for recovery in demyelinating diseases.

\section{References}

Bieber AJ, Kerr S, Rodriguez M (2003) Efficient central nervous system remyelination requires T cells. Ann Neurol 53:680-684.

Bligh EG, Dyer WJ (1959) A rapid method of total lipid extraction and purification. Can J Biochem Physiol 37:911-917.

Bogie JF, Grajchen E, Wouters E, Garcia Corrales A, Dierckx T, Vanherle S, Mailleux J, Gervois P, Wolfs E, Dehairs J, Van Broeckhoven J, Bowman AP, Lambrichts I, Gustafsson JA, Remaley AT, Mulder M, Swinnen JV, Haidar M, Ellis SR, Ntambi JM, et al. (2020) Stearoyl-CoA desaturase-1 impairs the reparative properties of macrophages and microglia in the brain. J Exp Med 217:e20191660.

Burgess M, Wicks K, Gardasevic M, Mace KA (2019) Cx3CR1 expression identifies distinct macrophage populations that contribute differentially to inflammation and repair. Immunohorizons 3:262-273.

Church JS, Milich LM, Lerch JK, Popovich PG, McTigue DM (2017) E6020, a synthetic TLR4 agonist, accelerates myelin debris clearance, Schwann cell infiltration, and remyelination in the rat spinal cord. Glia 65:883899.

Classen A, Lloberas J, Celada A (2009) Macrophage activation: classical versus alternative. Methods Mol Biol 531:29-43.

Cooper PH, Mayer P, Baggiolini M (1984) Stimulation of phagocytosis in bone marrow-derived mouse macrophages by bacterial lipopolysaccharide: correlation with biochemical and functional parameters. J Immunol 133:913-922.

Cunha MI, Su M, Cantuti-Castelvetri L, Muller SA, Schifferer M, Djannatian M, Alexopoulos I, van der Meer F, Winkler A, van Ham TJ, Schmid B, Lichtenthaler SF, Stadelmann C, Simons M (2020) Pro-inflammatory activation following demyelination is required for myelin clearance and oligodendrogenesis. J Exp Med 217:e20191390.

Dombrowski Y, O'Hagan T, Dittmer M, Penalva R, Mayoral SR, Bankhead P, Fleville S, Eleftheriadis G, Zhao C, Naughton M, Hassan R, Moffat J, Falconer J, Boyd A, Hamilton P, Allen IV, Kissenpfennig A, Moynagh PN, Evergren E, Perbal B, et al. (2017) Regulatory T cells promote myelin regeneration in the central nervous system. Nat Neurosci 20:674-680.

Doring A, Sloka S, Lau L, Mishra M, van Minnen J, Zhang X, Kinniburgh D, Rivest S, Yong VW (2015) Stimulation of monocytes, macrophages, and microglia by amphotericin B and macrophage colony-stimulating factor promotes remyelination. J Neurosci 35:1136-1148.
Durafourt BA, Moore CS, Zammit DA, Johnson TA, Zaguia F, Guiot MC, Bar-Or A, Antel JP (2012) Comparison of polarization properties of human adult microglia and blood-derived macrophages. Glia 60:717727.

Eichenfield DZ, Troutman TD, Link VM, Lam MT, Cho H, Gosselin D, Spann NJ, Lesch HP, Tao J, Muto J, Gallo RL, Evans RM, Glass CK (2016) Tissue damage drives co-localization of NF-kappaB, Smad3, and Nrf2 to direct Rev-erb sensitive wound repair in mouse macrophages. Elife 5:e13024.

El Waly B, Buttigieg E, Karakus C, Brustlein S, Debarbieux F (2020) Longitudinal intravital microscopy reveals axon degeneration concomitant with inflammatory cell infiltration in an LPC model of demyelination. Front Cell Neurosci 14:165.

Fabian RH, Rea HC (1993) Neuronal toxicity by macrophages in mixed brain cell culture is augmented by antineuronal IgG and dependent upon nitric oxide synthesis. J Neuroimmunol 44:95-102.

Fenn AM, Hall JC, Gensel JC, Popovich PG, Godbout JP (2014) IL-4 signaling drives a unique arginase ${ }^{+} / \mathrm{IL}^{-}$beta $^{+}$microglia phenotype and recruits macrophages to the inflammatory CNS: consequences of agerelated deficits in IL-4Ralpha after traumatic spinal cord injury. J Neurosci 34:8904-8917.

Foote AK, Blakemore WF (2005) Inflammation stimulates remyelination in areas of chronic demyelination. Brain 128:528-539.

Francos-Quijorna I, Amo-Aparicio J, Martinez-Muriana A, López-Vales R (2016) IL-4 drives microglia and macrophages toward a phenotype conducive for tissue repair and functional recovery after spinal cord injury. Glia 64:2079-2092.

Goldstein EZ, Church JS, Hesp ZC, Popovich PG, McTigue DM (2016) A silver lining of neuroinflammation: beneficial effects on myelination. Exp Neurol 283:550-559.

Guerrero BL, Sicotte NL (2020) Microglia in multiple sclerosis: friend or foe? Front Immunol 11:374.

Hauben E, Butovsky O, Nevo U, Yoles E, Moalem G, Agranov E, Mor F, Leibowitz-Amit R, Pevsner E, Akselrod S, Neeman M, Cohen IR, Schwartz M (2000) Passive or active immunization with myelin basic protein promotes recovery from spinal cord contusion. J Neurosci 20:6421-6430

Heinz S, Benner C, Spann N, Bertolino E, Lin YC, Laslo P, Cheng JX, Murre C, Singh H, Glass CK (2010) Simple combinations of lineage-determining transcription factors prime cis-regulatory elements required for macrophage and B cell identities. Mol Cell 38:576-589.

Huang JK, Ferrari CC, Monteiro de Castro G, Lafont D, Zhao C, Zaratin P, Pouly S, Greco B, Franklin RJ (2012) Accelerated axonal loss following acute CNS demyelination in mice lacking protein tyrosine phosphatase receptor type Z. Am J Pathol 181:1518-1523.

Ichihara Y, Doi T, Ryu Y, Nagao M, Sawada Y, Ogata T (2017) Oligodendrocyte progenitor cells directly utilize lactate for promoting cell cycling and differentiation. J Cell Physiol 232:986-995.

Jensen SK, Michaels NJ, Ilyntskyy S, Keough MB, Kovalchuk O, Yong VW (2018) Multimodal enhancement of remyelination by exercise with a pivotal role for oligodendroglial PGC1alpha. Cell Rep 24:3167-3179.

Jha AK, Huang SC, Sergushichev A, Lampropoulou V, Ivanova Y, Loginicheva E, Chmielewski K, Stewart KM, Ashall J, Everts B, Pearce EJ, Driggers EM, Artyomov MN (2015) Network integration of parallel metabolic and transcriptional data reveals metabolic modules that regulate macrophage polarization. Immunity 42:419-430.

Keough MB, Rogers JA, Zhang P, Jensen SK, Stephenson EL, Chen T, Hurlbert MG, Lau LW, Rawji KS, Plemel JR, Koch M, Ling CC, Yong VW (2016) An inhibitor of chondroitin sulfate proteoglycan synthesis promotes central nervous system remyelination. Nat Commun 7:11312.

Kierdorf K, Masuda T, Jordão MJ, Prinz M (2019) Macrophages at CNS interfaces: ontogeny and function in health and disease. Nat Rev Neurosci 20:547-562.

Kigerl KA, Gensel JC, Ankeny DP, Alexander JK, Donnelly DJ, Popovich PG (2009) Identification of two distinct macrophage subsets with divergent effects causing either neurotoxicity or regeneration in the injured mouse spinal cord. J Neurosci 29:13435-13444.

Kim JS, Kolesnikov M, Peled-Hajaj S, Scheyltjens I, Xia Y, Trzebanski S, Haimon Z, Shemer A, Lubart A, Van Hove H, Chappell-Maor L, BouraHalfon S, Movahedi K, Blinder P, Jung S (2021) A binary Cre transgenic approach dissects microglia and CNS border-associated macrophages. Immunity 54:176-190.e7. 
Kipnis J (2016) Multifaceted interactions between adaptive immunity and the central nervous system. Science 353:766-771.

Komiya H, Takeuchi H, Ogawa Y, Hatooka Y, Takahashi K, Katsumoto A, Kubota S, Nakamura H, Kunii M, Tada M, Doi H, Tanaka F (2020) CCR2 is localized in microglia and neurons, as well as infiltrating monocytes, in the lumbar spinal cord of ALS mice. Mol Brain 13:64.

Kotter MR, Zhao C, van Rooijen N, Franklin RJ (2005) Macrophage-depletion induced impairment of experimental CNS remyelination is associated with a reduced oligodendrocyte progenitor cell response and altered growth factor expression. Neurobiol Dis 18:166-175.

Langmead B, Salzberg SL (2012) Fast gapped-read alignment with Bowtie 2. Nat Methods 9:357-359.

Larsen PH, Yong VW (2004) The expression of matrix metalloproteinase-12 by oligodendrocytes regulates their maturation and morphological differentiation. J Neurosci 24:7597-7603.

Larsen PH, Wells JE, Stallcup WB, Opdenakker G, Yong VW (2003) Matrix metalloproteinase- 9 facilitates remyelination in part by processing the inhibitory NG2 proteoglycan. J Neurosci 23:11127-11135.

Laskin DL, Pendino KJ (1995) Macrophages and inflammatory mediators in tissue injury. Annu Rev Pharmacol Toxicol 35:655-677.

Lau LW, Keough MB, Haylock-Jacobs S, Cua R, Doring A, Sloka S, Stirling DP, Rivest S, Yong VW (2012) Chondroitin sulfate proteoglycans in demyelinated lesions impair remyelination. Ann Neurol 72:419-432.

Lee DC, Rizer J, Selenica ML, Reid P, Kraft C, Johnson A, Blair L, Gordon MN, Dickey CA, Morgan D (2010) LPS-induced inflammation exacerbates phospho-tau pathology in rTg4510 mice. J Neuroinflammation $7: 56$.

Lemarchant S, Pruvost M, Montaner J, Emery E, Vivien D, Kanninen K, Koistinaho J (2013) ADAMTS proteoglycanases in the physiological and pathological central nervous system. J Neuroinflammation 10:133.

Li J, Ramenaden ER, Peng J, Koito H, Volpe JJ, Rosenberg PA (2008) Tumor necrosis factor alpha mediates lipopolysaccharide-induced microglial toxicity to developing oligodendrocytes when astrocytes are present. J Neurosci 28:5321-5330.

Lloyd AF, Miron VE (2019) The pro-remyelination properties of microglia in the central nervous system. Nat Rev Neurol 15:447-458.

Lloyd AF, Davies CL, Holloway RK, Labrak Y, Ireland G, Carradori D, Dillenburg A, Borger E, Soong D, Richardson JC, Kuhlmann T, Williams A, Pollard JW, des Rieux A, Priller J, Miron VE (2019) Central nervous system regeneration is driven by microglia necroptosis and repopulation. Nat Neurosci 22:1046-1052.

Locatelli G, Theodorou D, Kendirli A, Jordao MJ, Staszewski O, Phulphagar K, Cantuti-Castelvetri L, Dagkalis A, Bessis A, Simons M, Meissner F, Prinz M, Kerschensteiner M (2018) Mononuclear phagocytes locally specify and adapt their phenotype in a multiple sclerosis model. Nat Neurosci 21:1196-1208.

Mantovani A, Sica A, Sozzani S, Allavena P, Vecchi A, Locati M (2004) The chemokine system in diverse forms of macrophage activation and polarization. Trends Immunol 25:677-686.

Masuda T, Amann L, Sankowski R, Staszewski O, Lenz M, D Errico P, Snaidero N, Costa Jordão MJ, Böttcher C, Kierdorf K, Jung S, Priller J, Misgeld T, Vlachos A, Meyer-Luehmann M, Knobeloch KP, Prinz M (2020) Novel Hexb-based tools for studying microglia in the CNS. Nat Immunol 21:802-815.

McMurran CE, Jones CA, Fitzgerald DC, Franklin RJ (2016) CNS remyelination and the innate immune system. Front Cell Dev Biol 4:38,

Mei F, Lehmann-Horn K, Shen YA, Rankin KA, Stebbins KJ, Lorrain DS, Pekarek K, A Sagan S, Xiao L, Teuscher C, von Büdingen HC, Wess J, Lawrence JJ, Green AJ, Fancy SP, Zamvil SS, Chan JR (2016) Accelerated remyelination during inflammatory demyelination prevents axonal loss and improves functional recovery. Elife 5:e18246.

Michaud JP, Halle M, Lampron A, Theriault P, Prefontaine P, Filali M, Tribout-Jover P, Lanteigne AM, Jodoin R, Cluff C, Brichard V, Palmantier R, Pilorget A, Larocque D, Rivest S (2013) Toll-like receptor 4 stimulation with the detoxified ligand monophosphoryl lipid A improves Alzheimer's disease-related pathology. Proc Natl Acad Sci USA 110:1941-1946.

Miron VE, Franklin RJ (2014) Macrophages and CNS remyelination. J Neurochem 130:165-171.

Miron VE, Boyd A, Zhao JW, Yuen TJ, Ruckh JM, Shadrach JL, van Wijngaarden P, Wagers AJ, Williams A, Franklin RJ, Ffrench-Constant C
(2013) M2 microglia and macrophages drive oligodendrocyte differentiation during CNS remyelination. Nat Neurosci 16:1211-1218.

Mishra MK, Wang J, Keough MB, Fan Y, Silva C, Sloka S, Hayardeny L, Bruck W, Yong VW (2014) Laquinimod reduces neuroaxonal injury through inhibiting microglial activation. Ann Clin Transl Neurol 1:409422.

Mylroie H, Dumont O, Bauer A, Thornton CC, Mackey J, Calay D, Hamdulay SS, Choo JR, Boyle JJ, Samarel AM, Randi AM, Evans PC, Mason JC (2015) PKCepsilon-CREB-Nrf2 signalling induces HO-1 in the vascular endothelium and enhances resistance to inflammation and apoptosis. Cardiovasc Res 106:509-519.

Plemel JR, Michaels NJ, Weishaupt N, Caprariello AV, Keough MB, Rogers JA, Yukseloglu A, Lim J, Patel VV, Rawji KS, Jensen SK, Teo W, Heyne B, Whitehead SN, Stys PK, Yong VW (2018) Mechanisms of lysophosphatidylcholine-induced demyelination: a primary lipid disrupting myelinopathy. Glia 66:327-347.

Plemel JR, Stratton JA, Michaels NJ, Rawji KS, Zhang E, Sinha S, Baaklini CS, Dong Y, Ho M, Thorburn K, Friedman TN, Jawad S, Silva C, Caprariello AV, Hoghooghi V, Yue J, Jaffer A, Lee K, Kerr BJ, Midha R, et al. (2020) Microglia response following acute demyelination is heterogeneous and limits infiltrating macrophage dispersion. Sci Adv 6:eaay6324.

Prinz M, Priller J (2017) The role of peripheral immune cells in the CNS in steady state and disease. Nat Neurosci 20:136-144.

Psachoulia K, Chamberlain KA, Heo D, Davis SE, Paskus JD, Nanescu SE, Dupree JL, Wynn TA, Huang JK (2016) IL4I1 augments CNS remyelination and axonal protection by modulating $\mathrm{T}$ cell driven inflammation. Brain 139:3121-3136.

Ransohoff RM (2016) How neuroinflammation contributes to neurodegeneration. Science 353:777-783.

Rapalino O, Lazarov-Spiegler O, Agranov E, Velan GJ, Yoles E, Fraidakis M, Solomon A, Gepstein R, Katz A, Belkin M, Hadani M, Schwartz M (1998) Implantation of stimulated homologous macrophages results in partial recovery of paraplegic rats. Nat Med 4:814-821.

Raposo C, Schwartz M (2014) Glial scar and immune cell involvement in tissue remodeling and repair following acute CNS injuries. Glia 62:18951904.

Rath M, Muller I, Kropf P, Closs EI, Munder M (2014) Metabolism via arginase or nitric oxide synthase: two competing arginine pathways in macrophages. Front Immunol 5:532.

Rawji KS, Kappen J, Tang W, Teo W, Plemel JR, Stys PK, Yong VW (2018) Deficient surveillance and phagocytic activity of myeloid cells within demyelinated lesions in aging mice visualized by ex vivo live multiphoton imaging. J Neurosci 38:1973-1988.

Rawji KS, Young AM, Ghosh T, Michaels NJ, Mirzaei R, Kappen J, Kolehmainen KL, Alaeiilkhchi N, Lozinski B, Mishra MK, Pu A, Tang W, Zein S, Kaushik DK, Keough MB, Plemel JR, Calvert F, Knights AJ, Gaffney DJ, Tetzlaff W, et al. (2020) Niacin-mediated rejuvenation of macrophage/microglia enhances remyelination of the aging central nervous system. Acta Neuropathol 139:893-909.

Rossi B, Constantin G, Zenaro E (2020) The emerging role of neutrophils in neurodegeneration. Immunobiology 225:151865.

Ruckh JM, Zhao JW, Shadrach JL, van Wijngaarden P, Rao TN, Wagers AJ, Franklin RJ (2012) Rejuvenation of regeneration in the aging central nervous system. Cell Stem Cell 10:96-103.

Scafidi J, Hammond TR, Scafidi S, Ritter J, Jablonska B, Roncal M, SzigetiBuck K, Coman D, Huang Y, McCarter RJ Jr, Hyder F, Horvath TL, Gallo V (2014) Intranasal epidermal growth factor treatment rescues neonatal brain injury. Nature 506:230-234.

Shechter R, London A, Varol C, Raposo C, Cusimano M, Yovel G, Rolls A, Mack M, Pluchino S, Martino G, Jung S, Schwartz M (2009) Infiltrating blood-derived macrophages are vital cells playing an anti-inflammatory role in recovery from spinal cord injury in mice. PLoS Med 6:e1000113.

Skihar V, Silva C, Chojnacki A, Doring A, Stallcup WB, Weiss S, Yong VW (2009) Promoting oligodendrogenesis and myelin repair using the multiple sclerosis medication glatiramer acetate. Proc Natl Acad Sci USA 106:17992-17997.

Spitzer M, Wildenhain J, Rappsilber J, Tyers M (2014) BoxPlotR: a web tool for generation of box plots. Nat Methods 11:121-122.

Stirling DP, Cummins K, Mishra M, Teo W, Yong VW, Stys P (2014) Tolllike receptor 2-mediated alternative activation of microglia is protective after spinal cord injury. Brain 137:707-723. 
Teixeira B, Bittencourt VC, Ferreira TB, Kasahara TM, Barros PO, Alvarenga R, Hygino J, Andrade RM, Andrade AF, Bento CA (2013) Low sensitivity to glucocorticoid inhibition of in vitro Th17-related cytokine production in multiple sclerosis patients is related to elevated plasma lipopolysaccharide levels. Clin Immunol 148:209-218.

Torres-Espin A, Forero J, Fenrich KK, Lucas-Osma AM, Krajacic A, Schmidt E, Vavrek R, Raposo P, Bennett DJ, Popovich PG, Fouad K (2018) Eliciting inflammation enables successful rehabilitative training in chronic spinal cord injury. Brain 141:1946-1962.

Triarhou LC, Herndon RM (1985) Effect of macrophage inactivation on the neuropathology of lysolecithin-induced demyelination. Br J Exp Pathol 66:293-301.

Turner RC, Naser ZJ, Lucke-Wold BP, Logsdon AF, Vangilder RL, Matsumoto RR, Huber JD, Rosen CL (2017) Single low-dose lipopolysaccharide preconditioning: neuroprotective against axonal injury and modulates glial cells. Neuroimmunol Neuroinflamm 4:6-15.

Vallieres N, Berard JL, David S, Lacroix S (2006) Systemic injections of lipopolysaccharide accelerates myelin phagocytosis during Wallerian degeneration in the injured mouse spinal cord. Glia 53:103-113.

Van den Bossche J, Baardman J, de Winther MP (2015) Metabolic characterization of polarized M1 and M2 bone marrow-derived macrophages using real-time extracellular flux analysis. J Vis Exp 105:53424.

Walsh JT, Hendrix S, Boato F, Smirnov I, Zheng J, Lukens JR, Gadani S, Hechler D, Gölz G, Rosenberger K, Kammertöns T, Vogt J, Vogelaar C, Siffrin V, Radjavi A, Fernandez-Castaneda A, Gaultier A, Gold R, Kanneganti TD, Nitsch R, et al. (2015) MHCII-independent CD4 ${ }^{+} \mathrm{T}^{\mathrm{T}}$ cells protect injured CNS neurons via IL-4. J Clin Invest 125:699-714.

Wee Yong V (2010) Inflammation in neurological disorders: a help or a hindrance? Neuroscientist 16:408-420.
Weljie AM, Dowlatabadi R, Miller BJ, Vogel HJ, Jirik FR (2007) An inflammatory arthritis-associated metabolite biomarker pattern revealed by $1 \mathrm{H}$ NMR spectroscopy. J Proteome Res 6:3456-3464.

Wynn TA, Vannella KM (2016) Macrophages in tissue repair, regeneration, and fibrosis. Immunity 44:450-462.

Xue J, Schmidt SV, Sander J, Draffehn A, Krebs W, Quester I, De Nardo D, Gohel TD, Emde M, Schmidleithner L, Ganesan H, Nino-Castro A, Mallmann MR, Labzin L, Theis H, Kraut M, Beyer M, Latz E, Freeman TC, Ulas T, et al. (2014) Transcriptome-based network analysis reveals a spectrum model of human macrophage activation. Immunity 40:274-288.

Yamasaki R, Lu H, Butovsky O, Ohno N, Rietsch AM, Cialic R, Wu PM, Doykan CE, Lin J, Cotleur AC, Kidd G, Zorlu MM, Sun N, Hu W, Liu L, Lee JC, Taylor SE, Uehlein L, Dixon D, Gu J, et al. (2014) Differential roles of microglia and monocytes in the inflamed central nervous system. J Exp Med 211:1533-1549.

Yin Y, Henzl MT, Lorber B, Nakazawa T, Thomas TT, Jiang F, Langer R, Benowitz LI (2006) Oncomodulin is a macrophage-derived signal for axon regeneration in retinal ganglion cells. Nat Neurosci 9:843-852.

Yong HY, Rawji KS, Ghorbani S, Xue M, Yong VW (2019) The benefits of neuroinflammation for the repair of the injured central nervous system. Cell Mol Immunol 16:540-546.

Zhou X, Wahane S, Friedl MS, Kluge M, Friedel CC, Avrampou K, Zachariou V, Guo L, Zhang B, He X, Friedel RH, Zou H (2020) Microglia and macrophages promote corralling, wound compaction and recovery after spinal cord injury via Plexin-B2. Nat Neurosci 23:337-350.

Ziv Y, Ron N, Butovsky O, Landa G, Sudai E, Greenberg N, Cohen H, Kipnis J, Schwartz M (2006) Immune cells contribute to the maintenance of neurogenesis and spatial learning abilities in adulthood. Nat Neurosci 9:268275 